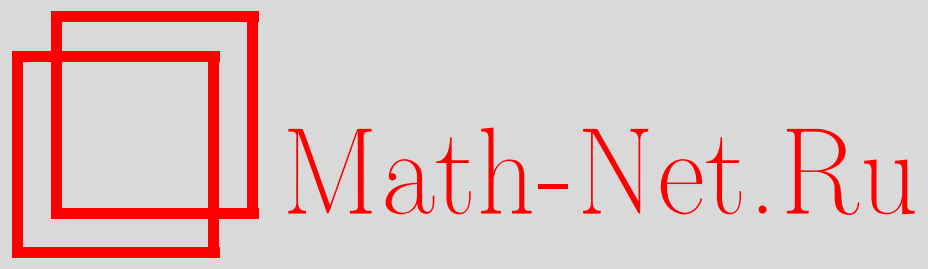

О. В. Огиевецкий, С. М. Хорошкин, Диагональная редукционная алгебра полной линейной алгебры Ли, Функи. анализ и его прил., 2010, том 44, выпуск $3,27-49$

DOI: https://doi.org/10.4213/faa2995

Использование Общероссийского математического портала MathNet.Ru подразумевает, что вы прочитали и согласны с пользовательским соглашением

http://www . mathnet.ru/rus/agreement

Параметры загрузки:

IP : 44.207 .124 .84

26 апреля 2023 г., 14:37:31

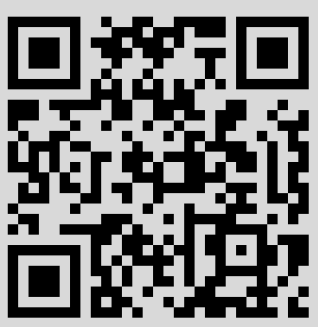


Функционалъный анализ и его приложения

2010, т. 44, вып. 3, с. 27-49

УДК $514.12+517.986$

\title{
Диагональная редукционная алгебра полной линейной алгебры Ли*
}

\author{
(c) 2010. О. В. ОГИЕВецКий, С. М. ХоРОшкин
}

\begin{abstract}
Исследованы общие свойства редукционных алгебр: алгоритмическая эффективность набора упорядочивающих соотношений и вопрос о том, над каким кольцом они определены. Сформулировано и доказано свойство стабилизации для редукционных

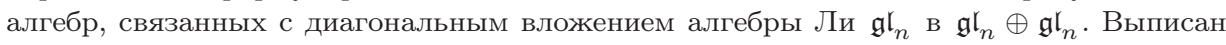
полный набор соотношений для этих редукционных алгебр.
\end{abstract}

\section{$\S 1$. Введение}

Редукционные алгебры были введены в работах [1], [7] для изучения представлений алгебры Ли с помощью ограничения представления на подалгебру.

Пусть $\mathfrak{g}$ - алгебра Ли, а $\mathfrak{k} \subset \mathfrak{g}$ - ее редуктивная подалгебра Ли, т. е. присоединенное действие $\mathfrak{k}$ в $\mathfrak{g}$ вполне приводимо (в частности, $\mathfrak{k}$ редуктивна). Предположим, что задано треугольное разложение

$$
\mathfrak{k}=\mathfrak{n}_{-}+\mathfrak{h}+\mathfrak{n}_{+} .
$$

Обозначим через $\mathrm{I}_{+}$левый идеал алгебры $\mathrm{A}:=\mathrm{U}(\mathfrak{g})$, порожденный элементами из $\mathfrak{n}_{+}, \mathrm{I}_{+}:=A \mathfrak{n}_{+}$. Редукционная алгебра $\mathrm{S}(\mathfrak{g}, \mathfrak{k})$, соответствующая паре $(\mathfrak{g}, \mathfrak{k})$, определяется как факторпространство $\operatorname{Norm}\left(\mathrm{I}_{+}\right) / \mathrm{I}_{+}$нормализатора идеала $\mathrm{I}_{+}$ по идеалу $\mathrm{I}_{+}$(следует иметь в виду, что обозначение $\mathrm{S}(\mathfrak{g}, \mathfrak{k})$ неполно: данные, необходимые для определения редукционной алгебры, включают в себя, помимо пары $(\mathfrak{g}, \mathfrak{k})$, треугольное разложение (1.1)). Пространство $\mathrm{S}(\mathfrak{g}, \mathfrak{k})$ обладает естественной структурой ассоциативной алгебры. По определению для всякого $\mathfrak{g}$-модуля $V$ пространство $V^{\mathfrak{n}_{+}}$векторов, аннулируемых элементами из $\mathfrak{n}_{+}$, есть $\mathrm{S}(\mathfrak{g}, \mathfrak{k})$-модуль. Если $V$ разлагается как $\mathfrak{k}$-модуль в прямую сумму неприводимых $\mathfrak{k}$-модулей $V_{i}$ с конечными кратностями, то $\mathfrak{g}$-модульная структура на $V$ однозначно восстанавливается по $\mathrm{S}(\mathfrak{g}, \mathfrak{k})$-модульной структуре на $V^{\mathfrak{n}_{+}}$.

Редукционная алгебра упрощается после локализации по мультипликативному множеству, порожденному элементами $h_{\gamma}+k$, где $\gamma$ пробегает множество корней алгебры $\mathfrak{k}, k \in \mathbb{Z}$; здесь $h_{\gamma}$ обозначает кокорень, соответствующий $\gamma$. Пусть $\overline{\mathrm{U}}(\mathfrak{h})$ - локализация по упомянутому мультипликативному множеству универсальной обертывающей алгебры $U(\mathfrak{h})$ подалгебры Картана $\mathfrak{h}$ алгебры Ли k. Локализованная редукционная алгебра Z $(\mathfrak{g}, \mathfrak{k})$ является алгеброй над коммутативным кольцом $\overline{\mathrm{U}}(\mathfrak{h})$; главная часть определяющих соотношений квадратична, однако соотношения могут содержать как линейные члены, так и члены

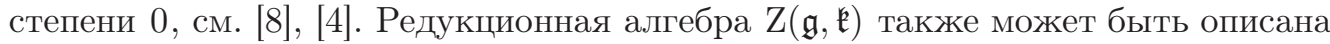
как (локализованное) пространство A/ $\left(\mathrm{An}_{+}+\mathfrak{n}_{-} \mathrm{A}\right)$ двойных смежных классов,

* Работа второго автора была поддержана грантом РФФИ 08-01-00667, совместным грантом CNRS-РФФИ 09-01-93106 и грантом 3036-2008-2 поддержки научных школ. Работа обоих авторов была поддержана ANR проектом GIMP No. ANR-05-BLAN-0029-01. 
снабженное умножением [4], определяемым при помощи экстремального проектора Ашеровой-Смирнова-Толстого [2].

Согласно общей теории редукционных алгебр [8], естественные образующие редукционной алгебры Z(g, k) удовлетворяют неоднородным квадратичным упорядочивающим соотношениям над полем частных $\mathscr{D}(\mathfrak{h})$ кольца $U(\mathfrak{h})$. При этом возникают два естественных вопроса: во-первых, определены ли эти упорядочивающие соотношения над меньшим кольцом $\overline{\mathrm{U}}(\mathfrak{h})$; во-вторых, можно ли использовать их для алгоритмического упорядочивания полиномиальных выражений в редукционной алгебре? В первой части работы мы даем положительные ответы на оба вопроса для любой редукционной алгебры $\mathrm{Z}(\mathfrak{g}, \mathfrak{k})$.

Вторая часть работы посвящена специальной редукционной задаче, в которой $\mathfrak{g}$ равна прямой сумме двух копий алгебры Ли $\mathfrak{g l}_{n}$, а $\mathfrak{k}$ представляет собой диагонально вложенную алгебру Ли $\mathfrak{g l}_{n}$. Соответствующую редукционную алгебру мы называем диагональной редукиионной алгеброй алгебры Ли $\mathfrak{g l}_{n}$ и обозначаем через $\mathrm{Z}_{n}$. Всякий конечномерный неприводимый модуль над $\mathfrak{g}=\mathfrak{g l}_{n} \oplus \mathfrak{g l}_{n}$ изоморфен тензорному произведению двух неприводимых $\mathfrak{g l}_{n}-$ модулей; ограничивая этот $\mathfrak{g}$-модуль на k, мы получаем разложение тензорного произведения в прямую сумму неприводимых $\mathfrak{g l}_{n}$-модулей. Один из главных результатов работы состоит в детальном описании редукционной алгебры $\mathrm{Z}_{n}$. Некоторые другие примеры и приложения диагональных редукционных алгебр приведены в работе [6].

Мы предъявляем полный список определяющих соотношений для естественных образующих алгебры $\mathrm{Z}_{n}$. Вывод этих соотношений, многократно использующий автоморфизмы Желобенко [4] редукционных алгебр, подробно представлен в работе [5]. В настоящей работе мы формулируем и доказываем для алгебр $\mathrm{Z}_{n}$ свойство стабилизации. Это свойство позволяет естественным образом продолжать соотношения с алгебры $\mathrm{Z}_{n}$ на алгебру $\mathrm{Z}_{n+1}\left(\mathrm{Z}_{n}\right.$ не есть подалгебра в $\left.\mathrm{Z}_{n+1}\right)$. Принцип стабилизации служит вторым важным ингредиентом для вывода определяющих соотношений в $\mathrm{Z}_{n}$.

Мы доказываем также, что наш список определяющих соотношений эквивалентен над $\overline{\mathrm{U}}(\mathfrak{h})$ списку канонических упорядочивающих соотношений. Доказательство несложно и сводится к анализу некоторых асимптотик, если ограничиться рассмотрением алгебр над полем $\mathscr{D}(\mathfrak{h})$. Доказательство эквивалентности над $\overline{\mathrm{U}}(\mathfrak{h})$ более тонко, оно использует стабилизацию и вычисление некоторых определителей Коши.

\section{§2. Редукционные алгебры, отвечающие редуктивной паре}

Пусть $\mathfrak{g}$ - конечномерная алгебра Ли и $\mathfrak{k} \subset \mathfrak{g}$ - ее редуктивная подалгебра. Предположим, что вложение $\mathfrak{k} \subset \mathfrak{g}$ также редуктивно, иными словами, присоединенное действие $\mathfrak{k}$ в $\mathfrak{g}$ вполне приводимо. Пусть $\mathfrak{p}$ есть $\mathrm{ad}_{\mathfrak{k}}$-инвариантное дополнение к k в $\mathfrak{g}$. Фиксируем треугольное разложение (1.1) алгебры Ли k; здесь $\mathfrak{h}$ - ее картановская подалгебра, а $\mathfrak{n}_{+}$и $\mathfrak{n}_{-}-$нильрадикалы двух противоположных борелевских подалгебр $\mathfrak{b}_{ \pm} \subset \mathfrak{k}$. Пусть $\Delta \in \mathfrak{h}^{*}-$ система корней алгебры Ли k. Подмножества множества $\Delta$, состоящие из положительных и отрицательных корней, обозначим через $\Delta_{+}$и $\Delta_{-}$соответственно. Пусть $\mathrm{Q}-$ 
решетка весов, Q $:=\left\{\gamma \in \mathfrak{h}^{*} \mid \gamma=\sum_{\alpha \in \Delta_{+}, n_{\alpha} \in \mathbb{Z}} n_{\alpha} \alpha\right\}$. Она содержит положительный конус

$$
\mathrm{Q}_{+}:=\left\{\gamma \in \mathfrak{h}^{*} \mid \gamma=\sum_{\alpha \in \Delta_{+}, n_{\alpha} \in \mathbb{Z}, n_{\alpha} \geqslant 0} n_{\alpha} \alpha\right\} .
$$

Для элементов $\lambda, \mu \in \mathfrak{h}^{*}$ запись

$$
\lambda>\mu
$$

означает, что $\lambda-\mu \in \mathrm{Q}_{+}$. Это частичный порядок в $\mathfrak{h}^{*}$.

Пусть W - группа Вейля системы корней $\Delta$. Пусть $\sigma_{1}, \ldots, \sigma_{r} \in \mathrm{W}$ - отражения в $\mathfrak{h}^{*}$, соответствующие простым корням $\alpha_{1}, \ldots, \alpha_{r}$. Мы используем также индуцированное действие группы Вейля W на векторном пространстве $\mathfrak{h}$. Оно определяется соотношением $\lambda(\sigma(H))=\sigma^{-1}(\lambda)(H)$ для любых $\sigma \in \mathrm{W}, H \in \mathfrak{h}$ и $\lambda \in \mathfrak{h}^{*}$. Мы предполагаем, что это действие продолжено до действия автоморфизмами алгебры Ли $\mathfrak{g}$ некоторой группы, накрывающей группу W. Точнее, заданы автоморфизмы $\hat{\sigma}_{i}: \mathfrak{g} \rightarrow \mathfrak{g}$, удовлетворяющие тем же соотношениям кос, что и $\sigma_{i}$; предполагается также, что эти автоморфизмы переводят подалгебры Ли $\mathfrak{h}$ и $\mathfrak{k}$ в себя и ограничения автоморфизмов $\hat{\sigma}_{i}$ и $\sigma_{i}$ на $\mathfrak{h}$ совпадают. Мы обозначаем теми же символами канонические продолжения отображений $\dot{\sigma}_{i}$ до автоморфизмов алгебры $\mathrm{U}(\mathfrak{g})$.

Пусть $\rho$ - полусумма положительных корней алгебры Ли k. Сдвинутое дейcmвие о группы W в векторном пространстве $\mathfrak{h}^{*}$ задается формулой

$$
\sigma \circ \lambda=\sigma(\lambda+\rho)-\rho .
$$

Рассматривая элементы коммутативной алгебры $U(\mathfrak{h})$ как полиномиальные функции на $\mathfrak{h}^{*}$, мы определяем с помощью формулы (2.3) действие о группы $\mathrm{W}$ и на этой алгебре. В частности, $(\sigma \circ H)(\lambda)=H\left(\sigma^{-1} \circ \lambda\right)$ для $H \in \mathfrak{h}$.

Для всякого $i=1, \ldots, r$ обозначим через $h_{\alpha_{i}}=\alpha_{i}^{\vee} \in \mathfrak{h}$ кокорень, соответствующий простому корню $\alpha_{i}$, так что значение $\alpha_{j}\left(H_{i}\right)$ равно $(i, j)$-элементу $a_{i j}$ матрицы Картана $a$ алгебры Ли k. Здесь $h_{\alpha_{i}}$ принадлежит полупростой части алгебры k. Пусть $e_{\alpha_{i}} \in \mathfrak{n}_{+}$и $e_{-\alpha_{i}} \in \mathfrak{n}_{-}-$образующие Шевалле алгебры $\mathfrak{k}$, соответствующие корням $\alpha_{i}$ и $-\alpha_{i}$, так что

$$
\left[e_{\alpha_{i}}, e_{-\alpha_{j}}\right]=\delta_{i j} h_{\alpha_{i}}, \quad\left[h_{\alpha_{i}}, e_{\alpha_{j}}\right]=a_{i j} e_{\alpha_{j}}, \quad\left[h_{\alpha_{i}}, e_{-\alpha_{j}}\right]=-a_{i j} e_{-\alpha_{j}} .
$$

Для каждого $\alpha \in \Delta$ пусть $h_{\alpha}=\alpha^{\vee} \in \mathfrak{h}$ - соответствующий кокорень. Обозначим через $\overline{\mathrm{U}}(\mathfrak{h})$ кольцо частных коммутативной алгебры $\mathrm{U}(\mathfrak{h})$ по отношению к мультипликативному множеству знаменателей, порожденному элементами

$$
\left\{h_{\alpha}+l \mid \alpha \in \Delta, l \in \mathbb{Z}\right\} .
$$

Элементы этого кольца можно также рассматривать как рациональные функции на векторном пространстве $\mathfrak{h}^{*}$. Элементы из $\mathrm{U}(\mathfrak{h}) \subset \overline{\mathrm{U}}(\mathfrak{h})$ тогда соответствуют полиномиальным функциям на $\mathfrak{h}^{*}$. Пусть $\overline{\mathrm{U}}(\mathfrak{k}) \subset \overline{\mathrm{A}}=\overline{\mathrm{U}}(\mathfrak{g})$ - кольца частных алгебр $\mathrm{U}(\mathfrak{k})$ и $\mathrm{A}=\mathrm{U}(\mathfrak{g})$ по отношению к множеству знаменателей (2.4). Эти кольца корректно определены, так как оба кольца $U(\mathfrak{k})$ и $U(\mathfrak{g})$ удовлетворяют условию Оре по отношению к (2.4). Поскольку отображения $\sigma_{i}$ сохраняют множество знаменателей (2.4), автоморфизмы $\sigma_{i}$ естественно продолжаются до автоморфизмов алгебры $\overline{\mathrm{A}}$.

Определим $\mathrm{Z}(\mathfrak{g}, \mathfrak{k})$ как пространство двойных смежных классов алгебры $\overline{\mathrm{A}}$ по ее левому идеалу $\overline{\mathrm{I}}_{+}:=\overline{\mathrm{A}} \mathfrak{n}_{+}$, порожденному элементами множества $\mathfrak{n}_{+}$, и ее 
правому идеалу $\overline{\mathrm{I}}_{-}:=\mathfrak{n}_{-} \overline{\mathrm{A}}$, порожденному элементами множества $\mathfrak{n}_{-}, \mathrm{Z}(\mathfrak{g}, \mathfrak{k}):=$ $\overline{\mathrm{A}} /\left(\overline{\mathrm{I}}_{+}+\overline{\mathrm{I}}_{-}\right)$. Операция умножения

$$
a \diamond b:=a P b
$$

превращает $\mathrm{Z}(\mathfrak{g}, \mathfrak{k})$ в ассоциативную алгебру. Здесь $P$ - экстремальный проектор [2] алгебры Ли k , соответствующий треугольному разложению (1.1). Мы называем $\mathrm{Z}(\mathfrak{g}, \mathfrak{k})$ редукиионной алгеброй пары $(\mathfrak{g}, \mathfrak{k})$. Соответствие $x \mapsto x \bmod \overline{\mathrm{I}}_{+}+$ $\overline{\mathrm{I}}_{-}$устанавливает гомоморфное вложение алгебры $\mathrm{S}(\mathfrak{g}, \mathfrak{k})$ (определение дано во введении) в $\mathrm{Z}(\mathfrak{g}, \mathfrak{k})$. Более того, локализация образа алгебры $\mathrm{S}(\mathfrak{g}, \mathfrak{k})$ по отношению к $\overline{\mathrm{U}}(\mathfrak{h})$ совпадает с Z $(\mathfrak{g}, \mathfrak{k})$. Отметим, что здесь мы используем определение алгебры $\mathrm{Z}(\mathfrak{g}, \mathfrak{k})$, отличное от приведенного во введении. Эквивалентность этих определений установлена в [4].

В алгебре $\mathrm{Z}(\mathfrak{g}, \mathfrak{k})$ можно задать действие автоморфизмов Желобенко [4]. Пусть $\check{\mathrm{q}}_{i}: \mathrm{Z}(\mathfrak{g}, \mathfrak{k}) \rightarrow \mathrm{Z}(\mathfrak{g}, \mathfrak{k})$ - автоморфизм Желобенко, соответствующий простому корню $\alpha_{i}, i=1, \ldots, r$. Он определяется следующим образом [4]. Сначала определим отображение $\check{q}_{i}: \mathrm{A} \rightarrow \overline{\mathrm{A}} / \overline{\mathrm{I}}_{+}$формулой

$$
\check{\mathrm{q}}_{i}(x):=\sum_{k \geqslant 0} \frac{(-1)^{k}}{k !} \hat{e}_{\alpha_{i}}^{k}\left(\hat{\sigma}_{i}(x)\right) e_{-\alpha_{i}}^{k} \prod_{j=1}^{k}\left(h_{\alpha_{i}}-j+1\right)^{-1} \bmod \overline{\mathrm{I}}_{+} .
$$

Здесь $\hat{x}$ обозначает присоединенное действие элемента $x$, так что $\hat{x}(y)=x y-y x$ для $x \in \mathfrak{k}$ и $y \in \overline{\mathrm{A}}$. Оператор $\check{\mathrm{q}}_{i}$ обладает следующим свойством:

$$
\check{\mathrm{q}}_{i}(h x)=\left(\sigma_{i} \circ h\right) \check{\mathrm{q}}_{i}(x)
$$

для всякого $x \in \mathrm{A}$ и $h \in \mathfrak{h}$; операция $\sigma \circ h$ определена в (2.3). Используя (2.7), мы можем продолжить $\check{\mathrm{q}}_{i}$ до отображения (обозначаемого тем же символом) $\check{\mathrm{q}}_{i}: \overline{\mathrm{A}} \rightarrow \overline{\mathrm{A}} / \overline{\mathrm{I}}_{+}$. А именно, для всякого $x \in \mathrm{A}$ и $\phi \in \overline{\mathrm{U}}(\mathfrak{h})$ полагаем $\check{\mathrm{q}}_{i}(\phi x)=\left(\sigma_{i} \circ \phi\right) \check{\mathrm{q}}_{i}(x)$. Можно показать, что $\check{\mathrm{q}}_{i}\left(\overline{\mathrm{I}}_{+}\right)=0$ и $\check{\mathrm{q}}_{i}\left(\overline{\mathrm{I}}_{-}\right) \subset\left(\overline{\mathrm{I}}_{-}+\overline{\mathrm{I}}_{+}\right) / \overline{\mathrm{I}}_{+}$, так что $\check{\mathrm{q}}_{i}$ можно интерпретировать как линейный оператор $\check{\mathrm{q}}_{i}: \mathrm{Z}(\mathfrak{g}, \mathfrak{k}) \rightarrow \mathrm{Z}(\mathfrak{g}, \mathfrak{k})$. Согласно [4], это отображение есть автоморфизм алгебр, удовлетворяющий (2.7). Операторы $\check{\mathrm{q}}_{i}$ удовлетворяют тем же соотношениям кос, что и $\sigma_{i}$. Имеет место следующая формула обращения [4]:

$$
\check{\mathrm{q}}_{i}^{2}(x)=\left(h_{\alpha_{i}}+1\right)^{-1} \hat{\sigma}_{i}^{2}(x)\left(h_{\alpha_{i}}+1\right), \quad x \in \mathrm{Z}(\mathfrak{g}, \mathfrak{k}) .
$$

Пусть, как и ранее, $\mathfrak{p}$ обозначает аd- инвариантное дополнение к $\mathfrak{k}$ в $\mathfrak{g}$. Выберем линейный базис $\left\{p_{K}\right\}$ пространства $\mathfrak{p}$ и снабдим его некоторым линейным порядком $\prec$. Для всякого $a \in \overline{\mathrm{A}}$ обозначим через $\widetilde{a}$ образ элемента $a$ в редукционной алгебре; в частности, $\widetilde{p}_{K}$ - это образ в $\mathrm{Z}(\mathfrak{g}, \mathfrak{k})$ базисного вектора $p_{K} \in \mathfrak{p}$.

В общей теории редукционных алгебр имеют место следующие утверждения (по поводу утверждений (a)-(c) см. [8]):

(а) Поскольку $\mathfrak{h}$ нормализует как $\mathfrak{n}_{+}$, так и $\mathfrak{n}_{-}$, алгебра $Z(\mathfrak{g}, \mathfrak{k})$ есть $\bar{U}(\mathfrak{h})$-бимодуль по отношению к умножению на элементы из $\bar{U}(\mathfrak{h})$. Она свободна и как левый, и как правый $\overline{\mathrm{U}}(\mathfrak{h})$-модуль. В качестве порождающего (над $\overline{\mathrm{U}}(\mathfrak{h}))$ подпространства можно взять проекцию на $\mathrm{Z}(\mathfrak{g}, \mathfrak{k})$ пространства $\mathrm{S}(\mathfrak{p})$ симметрических тензоров в пространстве $\mathfrak{p}$, т. е. подпространство пространства $\mathrm{Z}(\mathfrak{g}, \mathfrak{k})$, образованное линейными комбинациями образов степеней $p^{\nu}$, где $p \in \mathfrak{p}$ и $\nu \geqslant 0$. 
(b) Полагая $\operatorname{deg}(\widetilde{X})=l$ для образа всякого произведения $X=p_{K_{1}} \cdots p_{K_{l}}$ $l$ элементов множества $\mathfrak{p}$ и $\operatorname{deg}(Y)=0$ для $Y \in \overline{\mathrm{U}}(\mathfrak{h})$, мы задаем на $\mathrm{Z}(\mathfrak{g}, \mathfrak{k})$ структуру фильтрованной алгебры. Подпространство $\mathrm{Z}(\mathfrak{g}, \mathfrak{k})^{(k)}$ элементов степени не более чем $k$ - свободный левый и свободный правый $\overline{\mathrm{U}}(\mathfrak{h})$-модуль с порождающим пространством, образованным линейными комбинациями образов степеней $p^{\nu}$, где $p \in \mathfrak{p}$ и $k \geqslant \nu \geqslant 0$.

(c) В дальнейшем мы будем использовать весовые упорядоченные базисы $\left\{p_{K}\right\}$, в которых каждый базисный элемент $p_{K}$ имеет определенный вес $\mu_{K}$,

$$
\left[h, p_{K}\right]=\mu_{K}(h) p_{K}
$$

для всех $h \in \mathfrak{h}$. При этом линейный порядок $\prec$ предполагается согласованным с частичным порядком < на пространстве $\mathfrak{h}^{*}$, см. (2.2); согласованность означает импликацию $\mu_{K}<\mu_{L} \Longrightarrow p_{K} \prec p_{L}$. В этом случае образы $\tilde{p}_{\bar{L}}$ мономов $(\bar{L}$ понимается как мультииндекс)

$$
p_{\bar{L}}:=p_{L_{1}}^{n_{1}} \cdots p_{L_{m}}^{n_{m}}, \quad p_{L_{1}} \prec \cdots \prec p_{L_{m}}, \quad k=n_{1}+\cdots+n_{m},
$$

в $\mathrm{Z}(\mathfrak{g}, \mathfrak{k})^{(k)}$ линейно независимы над $\overline{\mathrm{U}}(\mathfrak{h})$ и их проекции на факторпространство $\mathrm{Z}(\mathfrak{g}, \mathfrak{k})^{(k)} / \mathrm{Z}(\mathfrak{g}, \mathfrak{k})^{(k-1)}$ образуют базис левого $\overline{\mathrm{U}}(\mathfrak{h})$-модуля $\mathrm{Z}(\mathfrak{g}, \mathfrak{k})^{(k)} / \mathrm{Z}(\mathfrak{g}, \mathfrak{k})^{(k-1)}$. Структурные константы алгебры $\mathrm{Z}(\mathfrak{g}, \mathfrak{k})$ в базисе $\left\{\widetilde{p}_{\bar{L}}\right\}$ лежат в кольце $\overline{\mathrm{U}}(\mathfrak{h})$.

Рассмотрим упорядоченный базис пространства $\mathfrak{k}+\mathfrak{p}$, начинающийся с базисных векторов пространства $\mathfrak{n}_{-}$и оканчивающийся базисными векторами пространства $\mathfrak{n}_{+}$. Алгебра А допускает базис Пуанкаре-Биркгофа-Витта (PBW), состоящий из упорядоченных мономов от элементов этого базиса. Из этого следует, что свойства мономов (2.10) в п. (с) имеют место без дополнительных ограничений на порядок $\prec$. Однако связь порядка $\prec$ с частичным порядком $<$ в $\mathfrak{h}^{*}$ будет существенной для большинства дальнейших утверждений.

(d) Алгебра $\mathrm{Z}(\mathfrak{g}, \mathfrak{k})$ - ассоциативная алгебра с единицей, порожденная подалгеброй $\overline{\mathrm{U}}(\mathfrak{h})$ и элементами $\widetilde{p}_{L}$, которые подчиняются весовым соотношениям (2.9) и упорядочивающим соотношениям

$$
\widetilde{p}_{I} \diamond \widetilde{p}_{J}=\sum_{K, L: p_{K} \preceq p_{L}} \mathrm{~B}_{I J K L} \widetilde{p}_{K} \diamond \widetilde{p}_{L}+\sum_{L} \mathrm{C}_{I J L} \widetilde{p}_{L}+\mathrm{D}_{I J}, \quad p_{I} \succ p_{J},
$$

где $\mathrm{B}_{I J K L}, \mathrm{C}_{I J L}$ и $\mathrm{D}_{I J}$ - некоторые элементы алгебры $\overline{\mathrm{U}}(\mathfrak{h})$.

Пусть $\mathscr{D}(\mathfrak{h})$ - поле частных кольца $\mathrm{U}(\mathfrak{h})$. В работе $[8, \S \S 4.2 .3-4.2 .4$ и 6.1.5] доказано, что редукционная алгебра порождена элементами $\widetilde{p}_{L}$ с определяющими упорядочивающими соотношениями (2.11) как алгебра над полем $\mathscr{D}(\mathfrak{h})$. Мы докажем, что утверждение (d) верно над меньшим кольцом $\overline{\mathrm{U}}(\mathfrak{h})$; иными словами, соотношения (2.11) определены над кольцом $\overline{\mathrm{U}}(\mathfrak{h})$ и элементы $\tilde{p}_{L}$ порождают над $\overline{\mathrm{U}}(\mathfrak{h})$ алгебру $\mathrm{Z}(\mathfrak{g}, \mathfrak{k})$.

Для этого покажем сначала, что структурные константы $\mathrm{B}_{I J K L}, \mathrm{C}_{I J L}$ и $\mathrm{D}_{I J}$ на самом деле принадлежат кольцу $\overline{\mathrm{U}}(\mathfrak{h})$. Это следует из факторизованной формулы для экстремального проектора $P$, см. [2]. В самом деле, разлагая произведение и упорядочивая слагаемые, мы представляем проектор $P$ в виде суммы слагаемых вида $\xi e_{-\gamma_{1}} \cdots e_{-\gamma_{m}} e_{\gamma_{1}^{\prime}} \cdots e_{\gamma_{m^{\prime}}^{\prime}}$, где $\xi \in \overline{\mathrm{U}}(\mathfrak{h})$, a $\gamma_{1}, \ldots, \gamma_{m}$ и $\gamma_{1}^{\prime}, \ldots, \gamma_{m^{\prime}}^{\prime}$ - положительные корни алгебры Ли k; при этом знаменатель элемента $\xi$ - произведение линейных сомножителей вида $h_{\gamma}+\rho\left(h_{\gamma}\right)+\ell$, где $\gamma-$ положительный корень алгебры Ли k и $\ell>0$ - положительное число. Далее мы вычисляем 
произведение $a \diamond b$ следующим образом. В слагаемом $a \xi e_{-\gamma_{1}} \cdots e_{-\gamma_{m}} e_{\gamma_{1}^{\prime}} \cdots e_{\gamma_{m^{\prime}}^{\prime}} b$ произведения $a \diamond b$ мы перемещаем $\xi$ и все множители вида $e_{-\gamma}$ налево через $a$, добавляя возникающие многократные коммутаторы с $a$; аналогично перемещаем все $e_{\gamma^{\prime}}$ направо через $b$. В результате получим равенство (напомним, что $\widetilde{a}$ означает образ элемента $a \in \mathrm{A}$ в редукционной алгебре)

$$
\widetilde{p}_{I} \diamond \widetilde{p}_{J}=\sum_{K, L} \mathrm{M}_{I J K L} \widehat{p_{K} p_{L}}
$$

где матрица М (однозначно определенная описанным выше способом вычисления) с элементами из $\overline{\mathrm{U}}(\mathfrak{h})$ имеет структуру треугольной матрицы с единицами на диагонали (верно даже более сильное утверждение: $\mathrm{M}_{I J K L} \neq 0 \Longrightarrow p_{I} \succeq$ $\left.p_{K}\right)$; знаменатели же элементов матрицы М имеют вид $h_{\gamma}+\rho\left(h_{\gamma}\right)+\pi\left(h_{\gamma}\right)+\bar{\ell}$, где $\pi$ - вес по отношению к $\mathfrak{h}$, соответствующий $p_{I}$, а $\ell-$ положительное целое число. Слагаемое $\pi\left(h_{\gamma}\right)$ появляется в начале вычисления $\widetilde{p}_{I} \diamond \widetilde{p}_{J}$, когда мы перемещаем $\xi \in \overline{\mathrm{U}}(\mathfrak{h})$ налево через $\widetilde{p}_{I}$; в процессе вычисления дальнейших многократных коммутаторов знаменатели более не меняются. Обратим формально соотношение (2.12), не обращая внимания на возможные алгебраические зависимости между $\widetilde{p_{I} p_{J}}$ или между $\widetilde{p}_{K} \diamond \widetilde{p}_{L}: \widetilde{p_{I} p_{J}}=\mathrm{M}_{I J K L}^{-1} \widetilde{p}_{K} \diamond \widetilde{p}_{L} ;$ при этом обратная матрица $\mathrm{M}^{-1}$ также треугольная с элементами из $\overline{\mathrm{U}}(\mathfrak{h})$ и единицами на диагонали. Поскольку определитель матрицы М равен единице, структура знаменателей элементов матрицы $\mathrm{M}^{-1}$ такая же, как и у элементов матрицы М. Коммутационные соотношения в $\mathrm{U}(\mathfrak{g}), p_{I} p_{J}=p_{J} p_{I}+\Upsilon, p_{I} \succ p_{J}, \Upsilon \in \mathfrak{g}$, принимают вид $\widetilde{p_{I} p_{J}}=\widetilde{p_{J} p_{I}}+\widetilde{\Upsilon}, \widetilde{\Upsilon} \in \mathfrak{p}+\mathfrak{h}$, в редукционной алгебре. Перепишем эти соотношения в виде правил упорядочивания для произведения $\diamond$, выражая проекции $\widetilde{p p}$ в терминах произведений $\widetilde{p} \diamond \widetilde{p}$ при помощи матрицы $\mathrm{M}^{-1}$ в обеих частях соотношений. При этом правая часть, будучи переписана в терминах умножения $\diamond$, будет содержать только упорядоченные слагаемые, левая же часть имеет вид $\widetilde{p}_{I} \diamond \widetilde{p}_{J}+\ldots$, где точками заменены слагаемые, содержащие произведения $\widetilde{p}_{I^{\prime}} \diamond \widetilde{p}_{J^{\prime}}, p_{I} \succ p_{I^{\prime}}$. Каждое такое слагаемое либо уже упорядочено, либо приводится к упорядоченной форме вида (2.11) (здесь мы используем индукцию по $I)$. Коэффициент при $\widetilde{p}_{I^{\prime}} \diamond \widetilde{p}_{J^{\prime}}$ принадлежит кольцу $\overline{\mathrm{U}}(\mathfrak{h})$; таким образом, упорядочивание произведений $\widetilde{p}_{I^{\prime}} \diamond \widetilde{p}_{J^{\prime}}$ может привести к появлению в (2.11) членов степени 1 или нуль с коэффициентами из $\overline{\mathrm{U}}(\mathfrak{h})$.

Аналогичным образом индукцией по степени фильтрации (она описана в п. (b)) доказывается, что алгебра $\mathrm{Z}(\mathfrak{g}, \mathfrak{k})$ порождена над $\overline{\mathrm{U}}(\mathfrak{h})$ элементами $\widetilde{p}_{L}$. Для этого рассмотрим весовой базис, описанный в утверждении (c), т. е. базис $\left\{\widetilde{p}_{\bar{L}}\right\}$ (здесь $\bar{L}$ - мультииндекс) свободного $\overline{\mathrm{U}}(\mathfrak{h})$-модуля $\mathrm{Z}(\mathfrak{g}, \mathfrak{k})^{(k)} / \mathrm{Z}(\mathfrak{g}, \mathfrak{k})^{(k-1)}$, образованного образами в $\mathrm{Z}(\mathfrak{g}, \mathfrak{k})$ произведений $p_{L_{1}}^{n_{1}} \cdots p_{L_{m}}^{n_{m}}$, где $p_{L_{1}} \prec \cdots \prec p_{L_{m}}$ и $k=n_{1}+\cdots+n_{m}$. Зададим на множестве базисных элементов линейный порядок $\prec$, согласованный с частичным порядком $<$ на $\mathfrak{h}^{*} ;$ согласованность понимается так же, как и для элементов $\widetilde{p}_{L}$ : [вес элемента $\left.\widetilde{p}_{\bar{K}}\right]<$ вес элемента $\left.\tilde{p}_{\bar{L}}\right] \Longrightarrow \tilde{p}_{\bar{K}} \prec \tilde{p}_{\bar{L}}$. Теми же, что и выше, аргументами, основанными на структуре проектора $P$, мы доказываем следующее обобщение формулы (2.12):

$$
\widetilde{p}_{I} \diamond \widetilde{p}_{\bar{J}}=\sum_{K, \bar{L}} \mathrm{M}_{I \bar{J} K \bar{L}} \widehat{p_{K} p_{\bar{L}}},
$$


где матрица M с элементами из $\overline{\mathrm{U}}(\mathfrak{h})$ опять имеет структуру треугольной матрицы с единицами на главной диагонали. Поэтому матрица М обратима и элементы обратной к ней матрицы $\mathrm{M}^{-1}$ принадлежат $\overline{\mathrm{U}}(\mathfrak{h})$. Из формулы $\widetilde{p_{I} p_{\bar{J}}}=$ $\mathrm{M}_{I \bar{J} K \bar{L}}^{-1} \widetilde{p}_{K} \diamond \tilde{p}_{\bar{L}}$ вытекает шаг индукции: подпространство $\mathrm{Z}(\mathfrak{g}, \mathfrak{k})^{(k+1)}$ порождено произведениями в $\mathrm{Z}(\mathfrak{g}, \mathfrak{k})$ элементов множества $\mathrm{Z}(\mathfrak{g}, \mathfrak{k})^{(1)}$.

Отметим, что до локализации алгебра $\mathrm{S}(\mathfrak{g}, \mathfrak{k})=\operatorname{Norm}\left(\mathrm{An} \mathfrak{n}_{+}\right) / A \mathfrak{n}_{+}$не порождается элементами степени 1. Подалгебра в $\mathrm{S}(\mathfrak{g}, \mathfrak{k})$, порожденная элементами степени 1 («step algebra»), была исходным объектом исследований Микельссона [7].

(е) Следующие мономы образуют базис левого $\overline{\mathrm{U}}(\mathfrak{h})$-модуля $\mathrm{Z}(\mathfrak{g}, \mathfrak{k})$ :

$$
\widetilde{p}_{I_{1}} \diamond \cdots \diamond \widetilde{p}_{I_{a}}, \quad p_{I_{1}} \preceq \cdots \preceq p_{I_{a}} .
$$

Докажем сначала несколько более тонкое утверждение.

Предложение 1. Всякое выражение в $\mathrm{Z}(\mathfrak{g}, \mathfrak{k})$ может быть записано в упорядоченной форме посредством многократного применения бормуль (2.11) в виде инструкиии «замените левую часть правой».

Доказательство. Для экономии места в процессе доказательства этого предложения мы будем иногда употреблять обозначение $I \prec J$ вместо $p_{I} \prec p_{J}$ (аналогичное соглашение используется для знаков $\preceq, \succ$ и $\succeq)$.

Рассмотрим однородную квадратичную часть соотношений (2.11):

$$
\widetilde{p}_{I_{1}} \diamond \widetilde{p}_{I_{2}}=\sum_{I_{1}^{\prime}, I_{2}^{\prime}: I_{1}^{\prime} \preceq I_{2}^{\prime}} \ldots \widetilde{p}_{I_{1}^{\prime}} \diamond \widetilde{p}_{I_{2}^{\prime}}, \quad I_{1} \succ I_{2},
$$

где точки заменяют коэффициенты из $\overline{\mathrm{U}}(\mathfrak{h})$. Обозначим через $\mathscr{I}\left(\widetilde{p}_{I_{1}} \diamond \widetilde{p}_{I_{2}}\right)$ правую часть равенства (2.15). Будем трактовать (2.15) как множество инструкций $\widetilde{p}_{I_{1}} \diamond \widetilde{p}_{I_{2}} \rightsquigarrow \mathscr{I}\left(\widetilde{p}_{I_{1}} \diamond \widetilde{p}_{I_{2}}\right)(\rightsquigarrow$ означает «заменить») в свободной алгебре с весовыми образующими $\tilde{p}_{I}$.

Докажем утверждение для кубического монома $\widetilde{p}_{I_{1}} \diamond \widetilde{p}_{I_{2}} \diamond \widetilde{p}_{I_{3}}$. Для такого монома мы можем применить инструкции (2.15) к $\widetilde{p}_{I_{1}} \diamond \widetilde{p}_{I_{2}}$, если $I_{1} \succ I_{2}$, и к $\widetilde{p}_{I_{2}} \diamond \widetilde{p}_{I_{3}}$, если $I_{2} \succ I_{3}$. Обозначим результаты через $\mathscr{I}_{12}\left(\widetilde{p}_{I_{1}} \diamond \widetilde{p}_{I_{2}} \diamond \widetilde{p}_{I_{3}}\right)$ и $\mathscr{I}_{23}\left(\widetilde{p}_{I_{1}} \diamond \widetilde{p}_{I_{2}} \diamond \widetilde{p}_{I_{3}}\right)$ соответственно.

Для всякого элемента $\psi \in \mathfrak{h}^{*}, \psi=\sum l_{i} \alpha_{i}$, где $\alpha_{i}$ - простые корни, положим $d(\psi):=\sum l_{i}$. Функция $d$ согласована с частичным порядком $<$ на $\mathfrak{h}^{*}$, т. е. $d(\alpha)<d(\beta)$, если $\alpha<\beta$. Обозначим тем же символом $d$ функцию на множестве индексов, нумерующих весовой базис пространства $\mathfrak{p}$, определенную равенством $d(I):=d\left(\mu_{I}\right)$, где $\mu_{I}-$ вес элемента $\widetilde{p}_{I}$.

Имеем равенство $d\left(I_{1}^{\prime}\right)+d\left(I_{2}^{\prime}\right)=d\left(I_{1}\right)+d\left(I_{2}\right)$ для всякого монома $\widetilde{p}_{I_{1}^{\prime}} \diamond \widetilde{p}_{I_{2}^{\prime}}$, появляющегося в правой части равенства (2.15) (заметим, что при этом разность $d\left(I_{1}\right)-d\left(I_{1}^{\prime}\right)$ есть целое число). Поскольку $I_{1} \succ I_{2}$ и $I_{1}^{\prime} \preceq I_{2}^{\prime}$, имеем неравенства $d\left(I_{1}\right) \geqslant d\left(I_{2}\right)$ и $d\left(I_{1}^{\prime}\right) \leqslant d\left(I_{2}^{\prime}\right)$; таким образом, $d\left(I_{1}^{\prime}\right) \leqslant d\left(\bar{I}_{1}\right)$ и $d\left(I_{2}^{\prime}\right) \geqslant d\left(I_{2}\right)$.

Каждому моному $\widetilde{p}_{I_{1}} \diamond \widetilde{p}_{I_{2}} \diamond \widetilde{p}_{I_{3}}$, т. е. упорядоченной тройке индексов $\left(I_{1}, I_{2}, I_{3}\right)$, сопоставим число $\mathfrak{d}\left(I_{1}, I_{2}, I_{3}\right):=2 d\left(I_{1}\right)+d\left(I_{2}\right)$. Применение инструкций упорядочивания $\mathscr{I}_{12}$ или $\mathscr{I}_{23} \mathrm{\kappa} \widetilde{p}_{I_{1}} \diamond \widetilde{p}_{I_{2}} \diamond \widetilde{p}_{I_{3}}$ не увеличивает значений функции $\mathfrak{d}$, иными словами, значение функции $\mathfrak{d}$ на каждом появившемся мономе не больше, чем $\mathfrak{d}\left(I_{1}, I_{2}, I_{3}\right)$. В самом деле, при замене $\widetilde{p}_{I_{1}} \diamond \widetilde{p}_{I_{2}}$ на $\widetilde{p}_{I_{1}^{\prime}} \diamond \widetilde{p}_{I_{2}^{\prime}}$ имеем $2 d\left(I_{1}^{\prime}\right)+d\left(I_{2}^{\prime}\right)=$ 
$d\left(I_{1}^{\prime}\right)+\left(d\left(I_{1}^{\prime}\right)+d\left(I_{2}^{\prime}\right)\right)=d\left(I_{1}^{\prime}\right)+\left(d\left(I_{1}\right)+d\left(I_{2}\right)\right) \leqslant d\left(I_{1}\right)+\left(d\left(I_{1}\right)+d\left(I_{2}\right)\right)=$ $2 d\left(I_{1}\right)+d\left(I_{2}\right)$; при замене $\widetilde{p}_{I_{2}} \diamond \widetilde{p}_{I_{3}}$ на $\widetilde{p}_{I_{2}^{\prime}} \diamond \widetilde{p}_{I_{3}^{\prime}}$ верны соотношения $d\left(I_{2}^{\prime}\right) \leqslant d\left(I_{2}\right)$ и $d\left(I_{1}^{\prime}\right)=d\left(I_{1}\right)$.

Для всякой линейной комбинации $X=\sum c_{I_{1} I_{2} I_{3}} \widetilde{p}_{I_{1}} \diamond \widetilde{p}_{I_{2}} \diamond \widetilde{p}_{I_{3}}$ кубических мономов с коэффициентами $c_{I_{1} I_{2} I_{3}} \in \overline{\mathrm{U}}(\mathfrak{h})$ обозначим тем же символом $\mathfrak{d}$ максимальное значение функции $\mathfrak{d}$ на мономах, встречающихся в $X$, т.е. $\mathfrak{d}(X):=$ $\max _{\left(I_{1}, I_{2}, I_{3}\right): c_{I_{1} I_{2} I_{3}} \neq 0} \mathfrak{d}\left(I_{1}, I_{2}, I_{3}\right)$.

Предположим, что утверждение неверно и существует кубический моном, который невозможно упорядочить, пользуясь инструкциями (2.15). Поскольку пространство $\mathfrak{k}+\mathfrak{p}$ конечномерно, множество значений функции $\mathfrak{d}$ на кубических мономах ограничено снизу. Поэтому минимальное значение $\mathfrak{d}_{\min }$ функции $\mathfrak{d}$ на множестве неупорядочиваемых кубических мономов конечно, $\mathfrak{d}_{\min }>-\infty$. Пусть $\widetilde{p}_{I_{1}} \diamond \widetilde{p}_{I_{2}} \diamond \widetilde{p}_{I_{3}}-$ моном, который нельзя упорядочить, причем $\mathfrak{d}\left(I_{1}, I_{2}, I_{3}\right)$ $=\mathfrak{d}_{\min }$. Применение инструкций упорядочивания (2.15) не может строго уменьшить значение функции $\mathfrak{d}$, ибо это противоречит минимальности значения $\mathfrak{d}\left(I_{1}, I_{2}, I_{3}\right)$. Поэтому среди появившихся мономов обязательно есть хотя бы один моном $\widetilde{p}_{I_{1}^{\prime}} \diamond \widetilde{p}_{I_{2}^{\prime}} \diamond \widetilde{p}_{I_{3}^{\prime}}$ с тем же значением функции $\mathfrak{d}$. Если моном $\widetilde{p}_{I_{1}^{\prime}} \diamond \widetilde{p}_{I_{2}^{\prime}} \diamond \widetilde{p}_{I_{3}^{\prime}}$ появился в инструкции $\mathscr{I}_{12}\left(\widetilde{p}_{I_{1}} \diamond \widetilde{p}_{I_{2}} \diamond \widetilde{p}_{I_{3}}\right)$, то $2 d\left(I_{1}^{\prime}\right)+d\left(I_{2}^{\prime}\right)=2 d\left(I_{1}\right)+d\left(I_{2}\right)$, $d\left(I_{1}\right) \geqslant d\left(I_{2}\right), d\left(I_{1}^{\prime}\right) \leqslant d\left(I_{2}^{\prime}\right)$ и $I_{3}^{\prime}=I_{3}$; общий вес при этом сохраняется, поэтому $d\left(I_{1}^{\prime}\right)=d\left(I_{1}\right)$ и $d\left(I_{2}^{\prime}\right)=d\left(I_{2}\right)$, откуда $d\left(I_{1}\right)=d\left(I_{2}\right)=d\left(I_{1}^{\prime}\right)=d\left(I_{2}^{\prime}\right)$. Если же моном $\widetilde{p}_{I_{1}^{\prime}} \diamond \widetilde{p}_{I_{2}^{\prime}} \diamond \widetilde{p}_{I_{3}^{\prime}}$ появился в инструкции $\mathscr{I}_{23}\left(\widetilde{p}_{I_{1}} \diamond \widetilde{p}_{I_{2}} \diamond \widetilde{p}_{I_{3}}\right)$, то $d\left(I_{2}\right) \geqslant d\left(I_{3}\right)$, $d\left(I_{2}^{\prime}\right) \leqslant d\left(I_{3}^{\prime}\right), d\left(I_{2}^{\prime}\right)=d\left(I_{2}\right)$ и $I_{1}^{\prime}=I_{1} ;$ аналогичные соображения приводят к равенствам $d\left(I_{2}\right)=d\left(I_{3}\right)=d\left(I_{2}^{\prime}\right)=d\left(I_{3}^{\prime}\right)$. Структура матрицы $\mathrm{M}$, определенной формулой (2.12), а также аргументы, использованные в доказательстве утверждения $(\mathrm{d})$, говорят о том, что при $d(I)=d(J)$ выражение $\mathscr{I}\left(\widetilde{p}_{I} \diamond \widetilde{p}_{J}\right)$ содержит в точности один моном $\widetilde{p}_{I^{\prime}} \diamond \widetilde{p}_{J^{\prime}}$ с условием $d\left(I^{\prime}\right)=d(I)$ и этот моном есть $\widetilde{p}_{J} \diamond \widetilde{p}_{I}$. Таким образом, с точностью до мономов, для которых значение функции $\mathfrak{d}$ меньше чем $\mathfrak{d}_{\min }$ (они могут быть упорядочены по нашему предположению), и коэффициентов из $\overline{\mathrm{U}}(\mathfrak{h})$, операция $\mathscr{I}_{12}, I_{1} \succ I_{2}$, совпадает с операцией $\widetilde{p}_{I_{1}} \diamond \widetilde{p}_{I_{2}} \rightsquigarrow \widetilde{p}_{I_{2}} \diamond \widetilde{p}_{I_{1}}$, а операция $\mathscr{I}_{23}, I_{2} \succ I_{3}$, совпадает с операцией $\widetilde{p}_{I_{2}} \diamond \widetilde{p}_{I_{3}} \rightsquigarrow \widetilde{p}_{I_{3}} \diamond \widetilde{p}_{I_{2}}$. Перестановки $(1,2)$ и $(2,3)$ соседей порождают все перестановки трех букв. Орбита $\widetilde{p}_{I_{1}} \diamond \widetilde{p}_{I_{2}} \diamond \widetilde{p}_{I_{3}}$ действия группы перестановок трех элементов $I_{1}, I_{2}$ и $I_{3}$ содержит упорядоченный моном, что приводит к противоречию.

Наличие в полных инструкциях (2.11) членов нулевой и первой степени приводит лишь к возникновению в процессе упорядочивания линейных или квадратичных членов. Поэтому всякий кубический полином может быть упорядочен и с помощью инструкций (2.11).

Более общо, сопоставим моному $X=\widetilde{p}_{I_{1}} \diamond \cdots \diamond \widetilde{p}_{I_{k}}$ произвольной степени $k$ число $\mathfrak{d}\left(I_{1}, \ldots, I_{k}\right):=(k-1) d\left(I_{1}\right)+(k-2) d\left(I_{2}\right)+\ldots+d\left(I_{k-1}\right)$ и в ситуации, когда значение этой величины минимально, заключим, что, с точностью до членов, в соответствующем смысле меньших $X$, инструкции по существу сводятся к перестановкам $(i, i+1)$ соседних индексов, порождающих всю группу перестановок множества из $k$ букв, так что упорядоченное выражение лежит в орбите элемента $X$ этой группы.

Доказательство утверждения (е). Согласно доказанному выше утверждению $(\mathrm{d})$, алгебра $\mathrm{Z}(\mathfrak{g}, \mathfrak{k})$ порождена элементами $\widetilde{p}_{I}$ и, благодаря форме $(2.11)$ соотношений, допускает фильтрацию по степени относительно умножения $\diamond$. 
Пусть $\mathrm{Z}(\mathfrak{g}, \mathfrak{k})^{(\diamond k)}$ - подпространство элементов степени не более $k$ по отношению к произведению $\diamond$. Из равенства $\widetilde{p}_{I_{1}} \diamond \ldots \diamond \widetilde{p}_{I_{k}}=\widetilde{p}_{I_{1}} P \widetilde{p}_{I_{2}} P \ldots P \widetilde{p}_{I_{k}}$ следует включение $\mathrm{Z}(\mathfrak{g}, \mathfrak{k})^{(\diamond k)} \subset \mathrm{Z}(\mathfrak{g}, \mathfrak{k})^{(k)}$. Обратное включение также справедливо, поскольку алгебра $\mathrm{Z}(\mathfrak{g}, \mathfrak{k})$ порождена элементами $\widetilde{p}_{I}$. Значит, эти две фильтрации совпадают.

Поэтому выражение ${\widetilde{p_{I_{1}} \cdots p_{I_{k}}}}, I_{1} \preceq \ldots \preceq I_{k}$, есть элемент пространства $\mathrm{Z}(\mathfrak{g}, \mathfrak{k})^{(\diamond k)}$ и, согласно только что доказанному предложению, может быть упорядочен. Мощности множеств $\left\{\widehat{p_{I_{1}} \cdots p_{I_{k}}} \mid I_{1} \preceq \cdots \preceq I_{k}\right\}$ и $\left\{\widetilde{p}_{I_{1}} \diamond \ldots \diamond \widetilde{p}_{I_{k}} \mid I_{1} \preceq\right.$ $\left.\cdots \preceq I_{k}\right\}$ равны, так что, согласно $(2.10)$, множество $\left\{\widetilde{p}_{I_{1}} \diamond \cdots \diamond \widetilde{p}_{I_{k}} \mid I_{1} \preceq \cdots \preceq I_{k}\right\}$ есть базис пространства $\mathrm{Z}(\mathfrak{g}, \mathfrak{k})^{(\diamond k)} / \mathrm{Z}(\mathfrak{g}, \mathfrak{k})^{(\diamond(k-1))}$.

Заметим, что для порядка, не согласованного с частичным порядком $<$ в $\mathfrak{h}^{*}$, упорядочивающие соотношения вида (2.11) могут существовать, однако утверждение (е) не обязательно верно. Например, упорядочивающие соотношения (2.11) могут быть выписаны для лексикографического порядка на образующих $z_{i j}$ и $t_{i}$ (считая, что $z_{i i}=t_{i}$ ) алгебры $\mathrm{Z}_{n}$, определенной в следующем параграфе, но при упорядочивании кубических мономов возникают циклы уже для $n=2$ (мы не приводим деталей; это результат явных вычислений).

\section{§3. Диагональная редукционная алгебра алгебры $Л и \mathfrak{g l}_{n}$}

Пусть $\mathfrak{g l}_{n}$ - алгебра Ли полной линейной группы $n$-мерного комплексного линейного пространства. Рассмотрим редуктивную пару $(\mathfrak{g}, \mathfrak{k})$, где $\mathfrak{g}=\mathfrak{g l}_{n} \oplus \mathfrak{g l}_{n}$ и $\mathfrak{k}=\mathfrak{g l}_{n}$ диагонально вложена в $\mathfrak{g l}_{n} \oplus \mathfrak{g l}_{n}$. Соответствующую редукционную алгебру мы называем диагональной редукиионной алгеброй и обозначаем символом $\mathrm{Z}_{n}$.

Фиксируем следующие обозначения для образующих так определенных алгебр $\mathfrak{g}$ и $\mathfrak{k}$. Пусть $E_{i j}^{(1)}$ и $E_{i j}^{(2)}, i, j=1, \ldots, n,-$ стандартные образующие двух копий алгебры Ли $\mathfrak{g l}_{n}$ внутри $\mathfrak{g l}_{n} \oplus \mathfrak{g l}_{n}$,

$$
\left[E_{i j}^{(p)}, E_{k l}^{(q)}\right]=\delta_{p q}\left(\delta_{j k} E_{i l}^{(p)}-\delta_{i l} E_{k j}^{(p)}\right),
$$

где $\delta_{s t}$ - символ Кронекера. Положим

$$
e_{i j}:=\frac{1}{2}\left(E_{i j}^{(1)}+E_{i j}^{(2)}\right), \quad E_{i j}:=\frac{1}{2}\left(E_{i j}^{(1)}-E_{i j}^{(2)}\right) .
$$

Элементы $e_{i j}$ линейно порождают диагонально вложенную алгебру Ли $\mathfrak{k} \simeq \mathfrak{g l}_{n}$, а элементы $E_{i j}$ линейно порождают присоединенный k-модуль $\mathfrak{p}$. Алгебра Ли $\mathfrak{k}$ и пространство $\mathfrak{p}$ составляют симметрическую пару, т. е. $[\mathfrak{k}, \mathfrak{k}] \subset \mathfrak{k},[\mathfrak{k}, \mathfrak{p}] \subset \mathfrak{p}$ и $[\mathfrak{p}, \mathfrak{p}] \subset \mathfrak{k}:$

$\left[e_{i j}, e_{k l}\right]=\delta_{j k} e_{i l}-\delta_{i l} e_{k j}, \quad\left[e_{i j}, E_{k l}\right]=\delta_{j k} E_{i l}-\delta_{i l} E_{k j}, \quad\left[E_{i j}, E_{k l}\right]=\delta_{j k} e_{i l}-\delta_{i l} e_{k j}$.

В дальнейшем мы обозначаем через $h_{a}$ элемент $e_{a a}$ подалгебры Картана $\mathfrak{h}$ алгебры Ли k $\in \mathfrak{g l}_{n} \oplus \mathfrak{g l}_{n}$, а через $h_{a b}$ - элемент $e_{a a}-e_{b b}$.

Пусть $\left\{\varepsilon_{a}\right\}$ - базис пространства $\mathfrak{h}^{*}$, двойственный к базису $\left\{h_{a}\right\}$ пространства $\mathfrak{h}, \varepsilon_{a}\left(h_{b}\right)=\delta_{a b}$. Мы также будем использовать корневые обозначения $h_{\alpha}$, $e_{\alpha}, e_{-\alpha}$ для элементов алгебры Ли k и $H_{\alpha}, E_{\alpha}, E_{-\alpha}$ для элементов пространства $\mathfrak{p}$. Подалгебра Ли $\mathfrak{n}_{+}$в треугольном разложении (1.1) порождена корне- 
выми векторами $e_{i j}$ c $i<j$; подалгебра Ли $\mathfrak{n}_{-}$порождена корневыми векторами $e_{i j}$ с $i>j$. Пусть $\mathfrak{b}_{+}$и $\mathfrak{b}_{-}-$соответствующие борелевские подалгебры, $\mathfrak{b}_{+}=\mathfrak{h} \oplus \mathfrak{n}_{+}, \mathfrak{b}_{-}=\mathfrak{h} \oplus \mathfrak{n}_{-}$. Система $\Delta_{+}$положительных корней алгебры Ли $\mathfrak{k}$ состоит из корней $\varepsilon_{i}-\varepsilon_{j}, i<j$, а система $\Delta_{-}-$из корней $\varepsilon_{i}-\varepsilon_{j}, i>j$.

Фиксируем следующее действие группы, накрывающей симметрическую группу $\mathrm{S}_{n}$ (группу Вейля диагональной подалгебры Ли k), автоморфизмами алгебры Ли $\mathfrak{g l}_{n} \oplus \mathfrak{g l}_{n}$ :

$$
\hat{\sigma}_{i}(x):=\operatorname{Ad}_{\exp \left(e_{i, i+1}\right)} \operatorname{Ad}_{\exp \left(-e_{i+1, i}\right)} \operatorname{Ad}_{\exp \left(e_{i, i+1}\right)}(x),
$$

так что $\hat{\sigma}_{i}\left(e_{k l}\right)=(-1)^{\delta_{i k}+\delta_{i l}} e_{\sigma_{i}(k) \sigma_{i}(l)}$ и $\dot{\sigma}_{i}\left(E_{k l}\right)=(-1)^{\delta_{i k}+\delta_{i l}} E_{\sigma_{i}(k) \sigma_{i}(l)}$. Здесь $\sigma_{i}=(i, i+1)$ - элементарная транспозиция в симметрической группе. Описанное действие группы, накрывающей группу $\mathrm{S}_{n}$, естественно продолжается до действия этой же группы автоморфизмами ассоциативной алгебры $\mathrm{A} \equiv \mathrm{A}_{n}:=$ $\mathrm{U}\left(\mathfrak{g l}_{n}\right) \otimes \mathrm{U}\left(\mathfrak{g l}_{n}\right)$. Ограничение этого действия на пространство $\mathfrak{h}$ совпадает с естественным действием $\sigma\left(h_{k}\right)=h_{\sigma(k)}, \sigma \in \mathrm{S}_{n}$, группы Вейля на подалгебре Картана.

Сдвинутое действие (2.3) группы Вейля на $\mathfrak{h}$ выглядит следующим образом:

$$
\sigma \circ h_{k}:=h_{\sigma(k)}+k-\sigma(k), \quad k=1, \ldots, n, \sigma \in \mathrm{S}_{n} .
$$

Оно превращается в обычное при переходе к переменным

$$
\stackrel{\circ}{h}_{k}:=h_{k}-k, \quad \stackrel{\circ}{h}_{i j}:=\stackrel{\circ}{h}_{i}-\stackrel{\circ}{h}_{j} ;
$$

т. е. $\sigma \circ \stackrel{\circ}{h}_{k}=\stackrel{\circ}{h}_{\sigma(k)}$ и $\sigma \circ \stackrel{\circ}{h}_{i j}=\stackrel{\circ}{h}_{\sigma(i) \sigma(j)}$ для всякого $\sigma \in \mathrm{S}_{n}$. Множество знаменателей, определяющих локализации $\overline{\mathrm{U}}(\mathfrak{h})$ и $\overline{\mathrm{A}}$, состоит из элементов

$$
h_{i j}+l, \quad l \in \mathbb{Z}, 1 \leqslant i<j \leqslant n .
$$

Мы выбираем множество векторов $E_{i j}, i, j=1, \ldots, n$, в качестве базиса пространства p. Вес элемента $E_{i j}$ равен $\varepsilon_{i}-\varepsilon_{j}$. Согласованность линейного порядка $\prec$ с частичным порядком $<$ на $\mathfrak{h}^{*}$ означает условие

$$
E_{i j} \prec E_{k l}, \quad \text { если } i-j>k-l .
$$

Порядок в каждом подмножестве $\left\{E_{i j} \mid i-j=a\right\}$, где $a$ фиксировано, может быть выбран произвольным. Например, можем считать, что

$$
E_{i j} \prec E_{k l}, \quad \text { если } i-j>k-l \text { или } i-j=k-l \text { и } i>k \text {. }
$$

Обозначим через $z_{i j}$ образ в $\mathrm{Z}_{n}$ элемента $E_{i j}$. Мы также будем использовать обозначения $t_{i}$ для элементов $z_{i i}$ и $t_{i j}:=t_{i}-t_{j}$ для элементов $z_{i i}-z_{j j}$. Порядок (3.8) индуцирует порядок на образующих $z_{i j}$ алгебры $\mathrm{Z}_{n}$ :

$$
z_{i j} \prec z_{k l} \Longleftrightarrow E_{i j} \prec E_{k l} .
$$

По утверждению $(\mathrm{d})$ из $\S 2$ существуют структурные константы $\mathrm{B}_{(a b),(c d),(i j),(k l)} \in$ $\overline{\mathrm{U}}(\mathfrak{h})$ и $\mathrm{D}_{(a b),(c d)} \in \overline{\mathrm{U}}(\mathfrak{h})$, такие, что для всех $a, b, c, d=1, \ldots, n$

$$
z_{a b} \diamond z_{c d}=\sum_{i, j, k, l: z_{i j} \preceq z_{k l}} \mathrm{~B}_{(a b),(c d),(i j),(k l)} z_{i j} \diamond z_{k l}+\mathrm{D}_{(a b),(c d)} .
$$

Линейные члены в правой части равенства (3.10) отсутствуют, поскольку пара $(\mathfrak{g}, \mathfrak{k})$ симметрическая. Соотношения (3.10) вместе с весовыми условиями

$$
\left[h, z_{a b}\right]=\left(\varepsilon_{a}-\varepsilon_{b}\right)(h) z_{a b}
$$


образуют полный набор определяющих соотношений алгебры $\mathrm{Z}_{n}$.

Структура знаменателей элементов матриц $\mathrm{M}$ и $\mathrm{M}^{-1}$, упомянутая выше в доказательстве соотношений (2.11), показывает, что для алгебры $\mathrm{Z}_{n}$ знаменатели структурных констант $\mathrm{B}_{(a b),(c d),(i j),(k l)}$ и $\mathrm{D}_{(a b),(c d)}$ суть произведения линейных множителей вида $\stackrel{\circ}{h}_{i j}+\ell, i<j$, где $\ell-$ целое число, $\ell \geqslant-1$. Это следует из того факта, что в нашей ситуации $\mathfrak{s l}_{2}$-подалгебра (диагональной алгебры $\left.\mathfrak{g l}_{n}\right)$, соответствующая произвольному положительному корню $\varepsilon_{i}-\varepsilon_{j}, i<j$, имеет в $\mathfrak{p}$ неприводимые представления размерности не выше 3 , так что числа $\ell$ в знаменателях проектора могут уменьшиться благодаря присутствию члена $(\pi, \gamma)$ не более чем на 2.

Антиинволюция Шевалле $\epsilon$ в $\mathrm{U}\left(\mathfrak{g l}_{n} \oplus \mathfrak{g l}_{n}\right), \epsilon\left(e_{i j}\right):=e_{j i}, \epsilon\left(E_{i j}\right):=E_{j i}$, индуцирует антиинволюцию $\epsilon$ в алгебре $\mathrm{Z}_{n}$ :

$$
\epsilon\left(z_{i j}\right)=z_{j i}, \quad \epsilon\left(h_{k}\right)=h_{k}
$$

Кроме того, внешний автоморфизм диаграммы Дынкина алгебры $\mathfrak{g l}_{n}$ определяет инволютивный автоморфизм $\omega$ алгебры $\mathrm{Z}_{n}$,

$$
\omega\left(z_{i j}\right)=(-1)^{i+j+1} z_{j^{\prime} i^{\prime}}, \quad \omega\left(h_{k}\right)=-h_{k^{\prime}},
$$

где $i^{\prime}=n+1-i$. Операции $\epsilon$ и $\omega$ коммутируют, $\epsilon \omega=\omega \epsilon$.

Центральные элементы подалгебры $\mathrm{U}\left(\mathfrak{g l}_{n}\right) \otimes 1 \subset \mathrm{A}$, порожденные $n$ элементами Казимира степеней $1, \ldots, n$, а также центральные элементы подалгебры $1 \otimes \mathrm{U}\left(\mathfrak{g l}_{n}\right) \subset \mathrm{A}$ проектируются в центральные элементы алгебры $\mathrm{Z}_{n}$. В частности, центральные элементы степени 1 проектируются в центральные элементы

$$
h_{1}+\cdots+h_{n} \quad \text { и } \quad t_{1}+\cdots+t_{n}
$$

алгебры $\mathrm{Z}_{n}$. Разность центральных элементов степени два проектируется в центральный элемент

$$
\sum_{i=1}^{n}\left(h_{i}-2 i\right) t_{i}
$$

алгебры $Z_{n}$. Образы остальных операторов Казимира более сложны.

3.1. Замена переменных. Мы будем использовать следующие элементы алгебры $\overline{\mathrm{U}}(\mathfrak{h})$ :

$$
A_{i j}:=\frac{\stackrel{\circ}{h}_{i j}}{\stackrel{\circ}{h}_{i j}-1}, \quad A_{i j}^{\prime}:=\frac{\stackrel{\circ}{h}_{i j}-1}{\stackrel{\circ}{h}_{i j}}, \quad B_{i j}:=\frac{\stackrel{\circ}{h}_{i j}-1}{\stackrel{\circ}{h}_{i j}-2}, \quad B_{i j}^{\prime}:=\frac{\stackrel{\circ}{h}_{i j}-2}{\stackrel{\circ}{h}_{i j}-1}, \quad C_{i j}^{\prime}:=\frac{\stackrel{\circ}{h}_{i j}-3}{\stackrel{\circ}{h}_{i j}-2},
$$

где переменные $\stackrel{\circ}{h}_{i j}$ определены равенствами (3.5). Заметим, что $A_{i j} A_{i j}^{\prime}=B_{i j} B_{i j}^{\prime}$ $=1$.

Определим элементы $\stackrel{\circ}{t}_{1}, \ldots, \stackrel{\circ}{t}_{n} \in \mathrm{Z}_{n}$ следующими соотношениями:

$$
\check{\circ}_{1}:=t_{1}, \quad \stackrel{\circ}{2}_{2}:=\check{\mathrm{q}}_{1}\left(t_{1}\right), \quad \stackrel{\circ}{t}_{3}:=\check{\mathrm{q}}_{2} \check{\mathrm{q}}_{1}\left(t_{1}\right), \quad \ldots, \quad \stackrel{\circ}{t}_{n}:=\check{\mathrm{q}}_{n-1} \cdots \check{\mathrm{q}}_{2} \check{\mathrm{q}}_{1}\left(t_{1}\right) .
$$


Используя (2.6), получаем соотношения

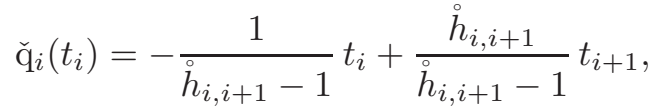

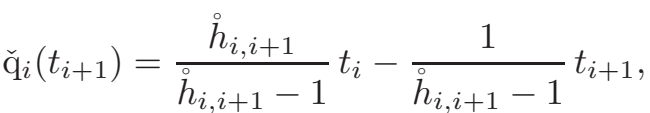

$$
\begin{aligned}
& \check{\mathrm{q}}_{i}\left(t_{k}\right)=t_{k}, \quad k \neq i, i+1,
\end{aligned}
$$

с помощью которых можно превратить соотношения (3.16) в линейную над кольцом $\overline{\mathrm{U}}(\mathfrak{h})$ замену переменных:

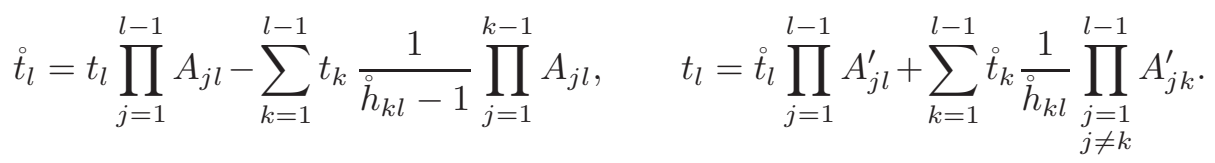

В терминах новых переменных $\stackrel{\circ}{i}_{i}$ второй из центральных элементов (3.14) выглядит так:

$$
\sum t_{i}=\sum \stackrel{\circ}{t}_{i} \prod_{a: a \neq i} \frac{\stackrel{\circ}{i a}_{i a}+1}{\circ_{i a}} .
$$

В дальнейшем мы используем понятие формул $c$ коэфбиииентами ограниченного роста. Это означает следующее. Пусть для каждого $n$ имеется набор формул (выражающих некоторое действие, соотношения еtс.) с коэффициентами из $\overline{\mathrm{U}}(\mathfrak{h})$. Мы говорим, что эти формулы имеют коэффициенты ограниченного роста, если степени числителей и знаменателей коэффициентов (в приведенной форме, после сокращения на общие множители) не растут с ростом $n$.

Например, приведенное ниже множество соотношений для $\mathrm{Z}_{n}$ имеет коэффициенты ограниченного роста, будучи записано в некотором специальном наборе образующих. В этом же смысле действие (3.17) имеет коэффициенты ограниченного роста, в то время как замена переменных (3.18) этим свойством не обладает.

3.2. Действие группы кос. Поскольку $\check{\mathrm{q}}_{i}^{2}(x)=x$ для всякого элемента $x$ нулевого веса, на пространстве нулевого веса действие группы кос сводится к действию ее факторгруппы - симметрической группы. Хотя коэффициенты в замене переменных (3.18) имеют неограниченный рост в смысле разд. 3.1, действие преобразований $\check{\mathrm{q}}_{i}$ на новые переменные $\check{t}_{i}$ имеет коэффициенты ограниченного роста. В самом деле, из определения (3.16) и соотношения $\check{q}_{i}\left(t_{1}\right)=t_{1}$ для всех $i>1$ следует, что

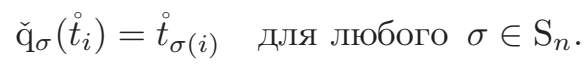

Действие автоморфизмов Желобенко на образующие $z_{k l}$ выглядит следующим образом:

$$
\begin{gathered}
\check{\mathrm{q}}_{i}\left(z_{i k}\right)=-z_{i+1, k} A_{i, i+1}, \quad \check{\mathrm{q}}_{i}\left(z_{k i}\right)=-z_{k, i+1}, \quad k \neq i, i+1, \\
\check{\mathrm{q}}_{i}\left(z_{i+1, k}\right)=z_{i, k}, \quad \check{\mathrm{q}}_{i}\left(z_{k, i+1}\right)=z_{k, i} A_{i, i+1}, \quad k \neq i, i+1, \\
\check{\mathrm{q}}_{i}\left(z_{i, i+1}\right)=-z_{i+1, i} A_{i, i+1} B_{i, i+1}, \quad \check{\mathrm{q}}_{i}\left(z_{i+1, i}\right)=-z_{i, i+1}, \\
\check{\mathrm{q}}_{i}\left(z_{j, k}\right)=z_{j, k}, \quad j, k \neq i, i+1 .
\end{gathered}
$$


Положим, как и раньше, $i^{\prime}=n+1-i$. Действие группы кос (3.20) согласовано с антиинволюцией $\epsilon$ и инволюцией $\omega$ (заметим, что $\left.\omega\left(\stackrel{\circ}{h}_{i j}\right)=\stackrel{\circ}{h}_{j^{\prime} i^{\prime}}\right)$, см. $(3.12)$ и (3.13), в следующем смысле:

$$
\epsilon \check{\mathrm{q}}_{i}=\check{\mathrm{q}}_{i}^{-1} \epsilon, \quad \omega \check{\mathrm{q}}_{i}=\check{\mathrm{q}}_{i^{\prime}-1} \omega .
$$

Пусть $w_{0}$ - элемент наибольшей длины группы Вейля алгебры Ли $\mathfrak{g l}_{n}$, т. е. симметрической группы $\mathrm{S}_{n}$. Отображение $\check{\mathrm{q}}_{w_{0}}^{2}$, подобно квадратам преобразований, соответствующих простым корням, см. (2.8), сводится к сопряжению некоторым элементом алгебры $\overline{\mathrm{U}}(\mathfrak{h})$. Прямая проверка показывает также, что

$$
\check{\mathrm{q}}_{w_{0}}\left(z_{i j}\right)=(-1)^{i+j} z_{i^{\prime} j^{\prime}} \prod_{a: a<i^{\prime}} A_{a i^{\prime}} \prod_{b: b>j^{\prime}} A_{j^{\prime} b}, \quad \check{\mathrm{q}}_{w_{0}}\left(\stackrel{\circ}{i}_{i}\right)=\check{\circ}_{i^{\prime}} .
$$

Из формулы (3.22) следует существование упорядочивающих соотношений на образующие $z_{i j}$ для порядка, обратного к порядку (3.8)-(3.9).

Следствие 2. Существуют такие $\mathrm{B}_{(a b),(c d),(i j),(k l)}^{\prime}, \mathrm{D}_{(a b),(c d)}^{\prime} \in \overline{\mathrm{U}}(\mathfrak{h})$, что для всех $z_{a b}$ и $z_{c d}$ верны соотношения

$$
z_{a b} \diamond z_{c d}=\sum_{i, j, k, l: z_{k l} \preceq z_{i j}} \mathrm{~B}_{(a b),(c d),(i j),(k l)}^{\prime} z_{i j} \diamond z_{k l}+\mathrm{D}_{(a b),(c d)}^{\prime} .
$$

В самом деле, применим преобразование $\check{q}_{w_{0}}$ к равенствам $(3.10)$, воспользовавшись формулой (3.22). Это приведет к соотношениям (3.23), поскольку подстановка $(i, j) \mapsto\left(i^{\prime}, j^{\prime}\right)$ обращает порядок $\prec$.

3.3. Определяющие соотношения. Для упрощения формул мы опускаем в этом разделе символ $\diamond$ для умножения в алгебре $\mathrm{Z}_{n}$. Это не должно привести к недоразумениям, поскольку в этом разделе мы не используем никаких других операций умножения в редукционной алгебре.

Каждое из выводимых нами соотношений имеет определенный вес, равный сумме двух корней. Из общих соображений верхняя оценка для числа членов в квадратичном соотношении веса $\lambda=\alpha+\beta$ есть число $|\lambda|$ квадратичных комбинаций $z_{\alpha^{\prime}} z_{\beta^{\prime}}$ с $\alpha^{\prime}+\beta^{\prime}=\lambda$. Имеется несколько типов весов соотношений, помимо тривиального $\lambda=2\left(\varepsilon_{i}-\varepsilon_{j}\right),|\lambda|=1$ :

1. $\lambda= \pm\left(2 \varepsilon_{i}-\varepsilon_{j}-\varepsilon_{k}\right)$, где $i, j$ и $k$ попарно различны. В этом случае $|\lambda|=2$.

2. $\lambda=\varepsilon_{i}-\varepsilon_{j}+\varepsilon_{k}-\varepsilon_{l}$ с попарно различными $i, j, k$ и $l$. В этом случае $|\lambda|=4$.

3. $\lambda=\varepsilon_{i}-\varepsilon_{j}, i \neq j$. Для монома $z_{\alpha^{\prime}} z_{\beta^{\prime}}$ имеется $2(n-2)$ возможностей (подтип 3а) с $\alpha^{\prime}=\varepsilon_{i}-\varepsilon_{k}, \beta^{\prime}=\varepsilon_{k}-\varepsilon_{j}$ или $\alpha^{\prime}=\varepsilon_{k}-\varepsilon_{j}, \beta^{\prime}=\varepsilon_{i}-\varepsilon_{k}$, где $k \neq i, j$, и $2 n$ возможностей (подтип $3 \mathrm{~b})$ с $\alpha^{\prime}=0, \beta^{\prime}=\varepsilon_{i}-\varepsilon_{j}$ или $\alpha^{\prime}=\varepsilon_{i}-\varepsilon_{j}, \beta^{\prime}=0$. Таким образом, $|\lambda|=4(n-1)$.

4. $\lambda=0$. Здесь имеется $n^{2}$ возможностей (подтип 4a) с $\alpha^{\prime}=0, \beta^{\prime}=0$ и $n(n-1)$ возможностей (подтип 4b) с $\alpha^{\prime}=\varepsilon_{i}-\varepsilon_{j}, \beta^{\prime}=\varepsilon_{j}-\varepsilon_{i}, i \neq j$. Таким образом, $|\lambda|=n(2 n-1)$.

Далее мы отдельно выписываем соотношения каждого типа и подтипа. Соотношения 1-го и 2-го типа имеют простой вид в исходных образующих $z_{i j}$. Для записи соотношений 3 -го и 4 -го типов удобно перенормировать образующие $z_{i j}$ с $i \neq j$. А именно, положим

$$
\stackrel{\check{z}}{i j}_{i j}=z_{i j} \prod_{k=1}^{i-1} A_{k i}
$$


В терминах образующих $\check{z}_{i j}$ формулы (3.20) для действия автоморфизмов $\check{q}_{i}$ приобретают вид

$$
\begin{aligned}
& \check{\mathrm{q}}_{i}\left(\check{z}_{i k}\right)=-\check{z}_{i+1, k}, \quad \check{\mathrm{q}}_{i}\left(\check{z}_{i+1, k}\right)=\check{z}_{i, k} A_{i+1, i}, \quad k \neq i, i+1, \\
& \check{\mathrm{q}}_{i}\left(\check{z}_{k i}\right)=-\check{\check{z}}_{k, i+1}, \quad \check{\mathrm{q}}_{i}\left(\check{\check{z}}_{k, i+1}\right)=\check{\check{z}}_{k, i} A_{i, i+1}=A_{i+1, i}^{\prime} \check{\check{z}}_{k, i}, \quad k \neq i, i+1, \\
& \check{\mathrm{q}}_{i}\left(\stackrel{\circ}{z}_{i, i+1}\right)=-A_{i+1, i}^{\prime} \stackrel{\circ}{z}_{i+1, i}, \quad \check{\mathrm{q}}_{i}\left(\stackrel{\circ}{z}_{i+1, i}\right)=-\check{\circ}_{i, i+1} A_{i+1, i}, \\
& \check{\mathrm{q}}_{i}\left(\check{z}_{j, k}\right)=\stackrel{\circ}{z}, k_{j,} \quad j, k \neq i, i+1 \text {. }
\end{aligned}
$$

Хотя в перенормировке (3.24) рост коэффициентов неограничен, действие группы кос по-прежнему имеет коэффициенты ограниченного роста.

1. Соотношения типа 1 имеют вид

$$
z_{i j} z_{i k}=z_{i k} z_{i j} A_{k j}, \quad z_{j i} z_{k i}=z_{k i} z_{j i} A_{k j}^{\prime} \quad \text { для } j<k, i \neq j, k .
$$

2. Введем обозначение $D_{i j k l}:=\stackrel{\circ}{h}_{i k}^{-1}-\stackrel{\circ}{h}_{j l}^{-1}$. Тогда для всяких четырех попарно различных индексов $i, j, k$ и $l$ выполнено следующее соотношение типа 2 :

$$
\begin{aligned}
{\left[z_{i j}, z_{k l}\right] } & =z_{k j} z_{i l} D_{i j k l}, & & i<k, j<l, \\
z_{i j} z_{k l}-z_{k l} z_{i j} A_{j l}^{\prime} A_{l j}^{\prime} & =z_{k j} z_{i l} D_{i j k l}, & & i<k, j>l .
\end{aligned}
$$

3а. Пусть $i \neq k \neq l \neq i$. Положим

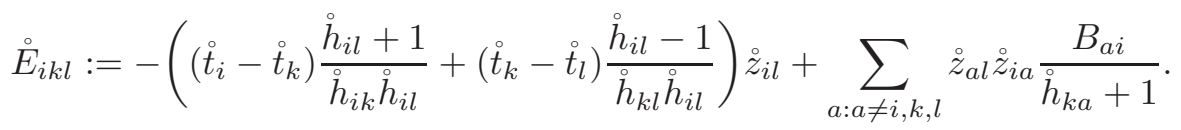

В этих обозначениях первая группа соотношений типа 3 имеет вид

$$
\begin{aligned}
& \stackrel{\circ}{z}_{i k} \stackrel{\circ}{z}_{k l} A_{i k}^{\prime}-\stackrel{\circ}{z}_{k l} \check{\circ}_{i k} B_{k i}=\stackrel{\circ}{E}_{i k l}, \quad i<k<l, \\
& \stackrel{\circ}{z}_{i k} \stackrel{\circ}{z}_{k l} A_{i k}^{\prime} A_{l k}^{\prime} B_{l k}-\stackrel{\circ}{z}_{k l} \stackrel{\circ}{z}_{i k} B_{k i}=\stackrel{\circ}{E}_{i k l}, \quad i<l<k,
\end{aligned}
$$

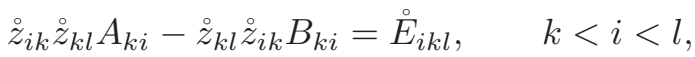



$$
\begin{aligned}
& \check{z}_{i k} \check{z}_{k l} A_{i k}^{\prime} A_{l k}^{\prime} B_{l k} A_{l i} B_{l i}^{\prime}-\check{\circ}_{k l} \check{z}_{i k} B_{k i}=\stackrel{\circ}{E}_{i k l}, \quad l<i<k, \\
& \check{z}_{i k} \check{z}_{k l} A_{k i} A_{l k}^{\prime} B_{l k} A_{l i} B_{l i}^{\prime}-\check{\check{z}}_{k l} \check{z}_{i k} B_{k i}=\stackrel{\circ}{E}_{i k l}, \quad l<k<i \text {. }
\end{aligned}
$$

Соотношения (3.27) можно переписать в более компактной форме с использованием обеих систем, $z_{i j}$ и $\check{z}_{i j}$, образующих алгебры. Пусть теперь

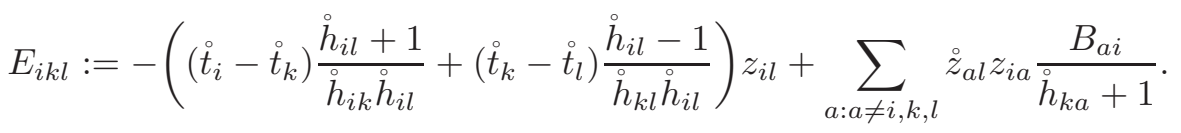

Тогда

$$
\begin{aligned}
& z_{i k} \stackrel{\circ}{k l}_{k l} A_{i k}^{\prime}-\stackrel{\circ}{z} k l_{z_{i k}} B_{k i}=E_{i k l}, \quad k<l, \\
& z_{i k} \stackrel{\circ}{z}_{k l} A_{i k}^{\prime} A_{l k}^{\prime} B_{l k}-\stackrel{\circ}{z}_{k l} z_{i k} B_{k i}=E_{i k l}, \quad l<k .
\end{aligned}
$$

Более того, дополнительная перенормировка $\stackrel{\circ}{z}_{k l}=\stackrel{\circ}{z}_{k l} B_{l k}$ при $k>l$ приводит к тому, что левая часть второй из формул (3.28) с точностью до общего множителя становится такой же, как левая часть первой строки этих же формул, а именно, равной $\left(z_{i k} \stackrel{\circ}{z}_{k l}^{\circ} A_{i k}^{\prime}-\stackrel{\circ}{z}_{k l}^{\circ} z_{i k} B_{k i}\right) A_{l k}^{\prime}$. 
3b. Пусть $l \neq j$. Вторая группа соотношений типа 3 такова:

$$
\begin{aligned}
& \stackrel{\circ}{z}_{i j} \stackrel{\circ}{i}_{i}=\stackrel{\circ}{t}_{i} \stackrel{z}{i j}_{i j}^{\prime}-\stackrel{\circ}{t}_{j} \stackrel{\circ}{z}_{i j} \frac{1}{\stackrel{\circ}{h}_{i j}+2}-\sum_{a: a \neq i, j}{\stackrel{\circ}{z_{a j}}}_{\stackrel{z}{z}_{i a}} \frac{1}{\stackrel{\circ}{h}_{i a}+2}, \\
& \stackrel{\circ}{z}_{i j} \stackrel{\circ}{t}_{j}=-\stackrel{\circ}{t}_{i} \stackrel{C}{z}_{i j} \frac{C_{j i}^{\prime}}{\stackrel{\circ}{h}_{i j}-1}+\stackrel{\circ}{t}_{j} \stackrel{\circ}{i j}_{i j} A_{i j} A_{j i}^{\prime} B_{j i}+\sum_{a: a \neq i, j} \stackrel{\circ}{z}_{a j} \stackrel{\circ}{z}_{i a} A_{i j} A_{j i}^{\prime} \frac{B_{a i}}{\stackrel{\circ}{h a}_{j}+1},
\end{aligned}
$$

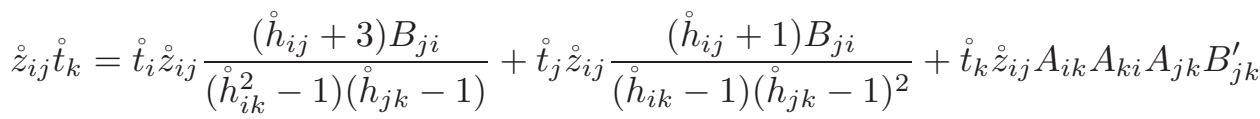

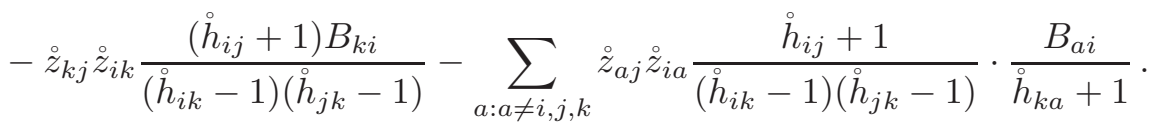

4а. Соотношения нулевого веса (тип 4) также разделяются на две группы. Соотношения из первой группы имеют вид

$$
\left[\stackrel{\circ}{i}_{i}, \stackrel{\circ}{j}_{j}=0\right.
$$

Отметим, что соотношения (3.30) справедливы для диагональной редукционной алгебры произвольной редуктивной алгебры Ли: образы образующих, соответствующих картановской подалгебре, коммутируют между собой.

4b. Соотношения второй группы типа 4 имеют вид (здесь $i \neq j$ )



На этом список соотношений заканчивается.

Обозначим через $\mathfrak{R}$ систему соотношений (3.25)-(3.27) и (3.29)-(3.31).

Теорема 3. Соотношения $\mathfrak{R}$ - определяющие соотношения для весовых образующих $z_{i j} u t_{i}$ алгебрь $\mathrm{Z}_{n}$. B частности, множество (3.10) упорядочивающих соотношений выводится над кольиом $\overline{\mathrm{U}}(\mathfrak{h})$ из соотношений $\mathfrak{R}(u$ эквивалентно им).

Вывод соотношений приведен в работе [5]; доказательство теоремы дано в $\$ 4$.

Соотношения (3.25)-(3.27) и (3.29)-(3.31) имеют коэффициенты ограниченного роста по отношению к образующим $\check{z}_{i j}$ и $\stackrel{\circ}{i}_{i}$; по отношению же к исходным образующим $z_{i j}$ и $t_{i}$ рост коэффициентов соотношений не ограничен. Мы склонны предполагать, что коэффициенты упорядочивающих соотношений (3.10) имеют неограниченный рост.

3.4. Стабилизация. Рассмотрим вложение алгебры Ли $\mathfrak{g l}_{n}$ в алгебру Ли $\mathfrak{g l}_{n+1}$, заданное соответствием $e_{i j} \mapsto e_{i j}, i, j=1, \ldots, n$, где элементы $e_{i j}$ в прообразе - это образующие алгебры Ли $\mathfrak{g l}_{n}$; в образе теми же символами $e_{i j}$ обозначены элементы алгебры $\mathfrak{g l}_{n+1}$. Аналогичное правило $E_{i j} \mapsto E_{i j}$ определяет вложение алгебры Ли $\mathfrak{g l}_{n} \oplus \mathfrak{g l}_{n}$ в алгебру $\mathfrak{g l}_{n+1} \oplus \mathfrak{g l}_{n+1}$ и обертывающей алгебры $\mathrm{A}_{n}=\mathrm{U}\left(\mathfrak{g l}_{n} \oplus \mathfrak{g l}_{n}\right)$ в $\mathrm{A}_{n+1}=\mathrm{U}\left(\mathfrak{g l}_{n+1} \oplus \mathfrak{g l}_{n+1}\right)$. При этом вложении нильпотентные подалгебры алгебры Ли $\mathfrak{g l}_{n}$ отображаются в соответствующие нильпотентные подалгебры алгебры Ли $\mathfrak{g l}_{n+1}$, так что определено вложение $\iota_{n}: Z_{n} \rightarrow Z_{n+1}$ соответствующих пространств двойных смежных классов. Однако отображение 
$\iota_{n}$ - не гомоморфизм алгебр, поскольку отображения умножения определены при помощи проекторов, которые различны для алгебр Ли $\mathfrak{g l}_{n}$ и $\mathfrak{g l}_{n+1}$.

Тем не менее между двумя умножениями имеется важная связь. А именно, пусть $\mathrm{V}_{n+1}$ - левый идеал алгебры $\mathrm{Z}_{n+1}$, порожденный элементами $z_{i, n+1}$, $i=1, \ldots, n$, a $\mathrm{V}_{n+1}^{\prime}$ - ее правый идеал, порожденный элементами $z_{n+1, i}, i=$ $1, \ldots, n$. Обозначим временно через $\diamond_{(n)}: \mathrm{Z}_{n} \otimes \mathrm{Z}_{n} \rightarrow \mathrm{Z}_{n}$ и $\diamond_{(n+1)}: \mathrm{Z}_{n+1} \otimes \mathrm{Z}_{n+1} \rightarrow$ $\mathrm{Z}_{n+1}$ операции умножения в $\mathrm{Z}_{n}$ и в $\mathrm{Z}_{n+1}$ (вместо используемого в других местах обозначения $\diamond$, см. $(2.5))$.

Пусть $\pi_{n+1}: \mathrm{Z}_{n+1} \rightarrow \mathrm{Z}_{n+1}$ - произвольный линейный оператор в $\mathrm{Z}_{n+1}$, проектирующий пространство $\mathrm{Z}_{n+1}$ на $\iota_{n}\left(\mathrm{Z}_{n}\right)$; предположим также, что либо идеал $\mathrm{V}_{n+1}$, либо идеал $\mathrm{V}_{n+1}^{\prime}$ лежит в ядре оператора $\pi_{n+1}$,

$$
\pi_{n+1}(x)=x, \quad x \in \iota_{n}\left(\mathrm{Z}_{n}\right), \quad \text { и } \quad \pi_{n+1}\left(\mathrm{~V}_{n+1}\right)=0 \quad \text { или } \quad \pi_{n+1}\left(\mathrm{~V}_{n+1}^{\prime}\right)=0 .
$$

Определим отображение $\tilde{\diamond}_{(n)}: \iota_{n}\left(\mathrm{Z}_{n}\right) \otimes \iota_{n}\left(\mathrm{Z}_{n}\right) \rightarrow \iota_{n}\left(\mathrm{Z}_{n}\right)$ как композицию,

$$
\tilde{\diamond}_{(n)}=\pi_{n+1} \diamond_{(n+1)} \text {. }
$$

Предложение 4. Имеет место следующее соотношение коммутативноcmu:

$$
\iota_{n} \diamond(n)=\tilde{\diamond}_{(n)}\left(\iota_{n} \otimes \iota_{n}\right) .
$$

Это утверждение можно уточнить: из его доказательства видно, что для всяких $i, j, k, l \leqslant n$ разность $\iota_{n}\left(z_{i j} \diamond_{(n)} z_{k l}\right)-z_{i j} \diamond_{(n+1)} z_{k l}$ в $\mathrm{Z}_{n+1}$ может быть представлена в виде $\sum_{a=1}^{n} z_{n+1, a} \diamond_{(n+1)} z_{i+k-j-l+a, n+1} \xi^{(a)}$, где $\xi^{(a)} \in \overline{\mathrm{U}}(\mathfrak{h})$.

Для доказательства предложения нам понадобится следующая лемма.

Лемма 5. Левый идеал алгебры $\mathrm{Z}_{n}$, порожденный всеми элементами $z_{i n}$, где $i=1, \ldots, n-1$, состоит из образов в $\mathrm{Z}_{n}$ сум⿻м $\sum_{i} X_{i} E_{i n}$, әде $X_{i} \in \overline{\mathrm{A}}$, $i=1, \ldots, n-1$.

Правый идеал алгебры $\mathrm{Z}_{n}$, порожденный элементами $z_{n i}, i=1, \ldots, n-1$, состоит из образов в $\mathrm{Z}_{n}$ сумм $\sum_{i} E_{n i} Y_{i}$, где $Y_{i} \in \overline{\mathrm{A}}, i=1, \ldots, n-1$.

Доказательство. Мы следуем аргументам, использованным при доказательстве соотношений (2.11). Представим проектор $P$ в виде суммы слагаемых вида $\xi e_{-\gamma_{1}} \cdots e_{-\gamma_{m}} e_{\gamma_{1}^{\prime}} \cdots e_{\gamma_{m^{\prime}}^{\prime}}$, где $\xi \in \overline{\mathrm{U}}(\mathfrak{h})$, а $\gamma_{1}, \ldots, \gamma_{m}$ и $\gamma_{1}^{\prime}, \ldots, \gamma_{m^{\prime}}^{\prime}$ - положительные корни алгебры k. Для всякого $\lambda \in Q_{+}$обозначим через $P_{\lambda}$ сумму описанных выше слагаемых с условием $\gamma_{1}+\cdots+\gamma_{m}=\gamma_{1}^{\prime}+\cdots+\gamma_{m^{\prime}}^{\prime}=\lambda$. Тогда $P=\sum_{\lambda \in Q_{+}} P_{\lambda}$. Для любых $X, Y \in \overline{\mathrm{A}}$ определим элемент $X \diamond_{\lambda} Y$ как образ элемента $X P_{\lambda} Y$ в редукционной алгебре, так что $X \diamond Y=\sum_{\lambda \in Q_{+}} X \diamond_{\lambda} Y$.

Для всякого $X \in \overline{\mathrm{A}}$ и $i<n$ рассмотрим произведение $X \diamond_{\lambda} z_{i n}$. Пусть $\lambda=\sum_{k=1}^{n} \lambda_{k} \varepsilon_{k}$. Произведение $X \diamond_{\lambda} z_{i n}$ равно нулю, если $\lambda_{n} \neq 0$. В самом деле, в этом случае в каждом слагаемом элемента $P_{\lambda}$ один из множителей $e_{\gamma_{k^{\prime}}^{\prime}}$ равен некоторому элементу $e_{j n}$. Мы можем упорядочить мономы в алгебре $\mathrm{U}\left(\mathfrak{n}_{+}\right)$таким образом, чтобы все элементы $e_{j n}$ стояли справа. Поскольку $\left[e_{j n}, E_{i n}\right]=0$, произведение $e_{j n} E_{i n}$ принадлежит левому идеалу $\overline{\mathrm{I}}_{+}$, так что $X \diamond_{\lambda} z_{i n}=0$ в алгебре $\mathrm{Z}_{n}$. Если же $\lambda_{n}=0$, то, используя PBW-аргументы, мы можем переписать элемент $P_{\lambda}$ в виде суммы мономов, составленных из образующих $e_{i j}$, $1 \leqslant i<j<n$, так что их присоединенное действие оставляет инвариантным пространство, порожденное всеми $E_{i n}$ с $i<n$. Таким образом, мы представили произведение $X \diamond_{\lambda} z_{i n}$ как образ суммы $\sum_{j} X_{j} E_{j n}$ с $X_{j} \in \overline{\mathrm{A}}$, где $j<n$. Значит, 
левый идеал, порожденный элементами $z_{i n}$, содержится в векторном пространстве образов в алгебре $\mathrm{Z}_{n}$ сумм $\sum_{i} X_{i} E_{i n}$. Более того, произведение $X \diamond z_{i n}$ есть образ элемента $X E_{i n}+\sum_{m<i} X^{(m)} E_{m n}$ для некоторых $X^{(m)}$. Теперь индукцией по $i$ мы доказываем обратное включение.

Вторая часть леммы доказывается аналогично.

Доказательство предложения 4. Достаточно доказать следующее утверждение. Пусть $X$ и $Y$ - (некоммутативные) полиномы от элементов $E_{i j}$ с $i, j \leqslant n$. Тогда произведение элементов $\widetilde{X}$ и $\widetilde{Y}$ в алгебре $\mathrm{Z}_{n+1}$ совпадает с образом в алгебре $\mathrm{Z}_{n+1}$ элемента $X P_{n} Y$, где $P_{n}$ - проектор для алгебры Ли $\mathfrak{g l}_{n}$, по модулю левого идеала в алгебре $\mathrm{Z}_{n+1}$, порожденного всеми элементами $z_{i, n+1}$, где $i \leqslant n$. Мы снова замечаем, что, благодаря структуре проектора, для любого веса $\lambda=\sum_{k} \lambda_{k} \varepsilon_{k}$ с $\lambda_{n+1}=0$ произведение $X \diamond_{\lambda} Y$, связанное с алгеброй $\mathfrak{g l}_{n}$, совпадает с произведением $X \diamond_{\lambda} Y$, связанным с алгеброй $\mathfrak{g l}_{n+1}$. Остается доказать, что для любых определенных выше полиномов $X$ и $Y$ элемент $\widetilde{X} \diamond_{\lambda} \widetilde{Y}$ принадлежит идеалу алгебры $\mathrm{Z}_{n+1}$, порожденному всеми элементами $z_{i, n+1} \mathrm{c}$ $i \leqslant n$, при условии, что $\lambda_{n+1} \neq 0$. Однако из весовых соображений следует, что для всякого веса $\lambda$ с $\lambda_{n+1} \neq 0$ элемент $\widetilde{X} \diamond_{\lambda} \widetilde{Y}$ может быть представлен как образ в алгебре $\mathrm{Z}_{n+1}$ суммы $\sum X_{i} Y_{i}$, в которой $(n+1)$-я компонента веса каждого из сомножителей $Y_{i}$ не равна нулю. Следовательно, каждый элемент $Y_{i}$ должен принадлежать левому идеалу, порожденному элементами $E_{j, n+1}, j=1, \ldots, n$. Для окончания доказательства предложения применяем лемму 5.

Часть предложения 4, относящаяся к идеалу $V_{n+1}^{\prime}$, доказывается аналогично.

Следствие 6. Коэффиииенты в соотношениях (3.25)-(3.27), (3.29)-(3.31) стабильны по отношению $\kappa$ определенным выше вложсениям алгебр $\mathrm{Z}_{n} \rightarrow$ $\mathrm{Z}_{n+1}$.

Стабильность коэффициентов понимается в следующем смысле. Пусть $\mathscr{R}$ соотношение в алгебре $\mathrm{Z}_{n+1}$ из нашего определяющего списка $\mathfrak{R}$, см. разд. 3.3. Предположим, что $\mathscr{R}$ не содержит членов с образующими $z_{i, n+1}, i=1, \ldots, n$, в качестве левого сомножителя. Тогда, если мы вычеркнем из $\mathscr{R}$ слагаемые, содержащие образующие $z_{i, n+1}, i=1, \ldots, n$, как правые сомножители (такое слагаемое обязательно содержит некоторую образующую $z_{n+1, j}, j=1, \ldots, n$, как левый сомножитель), мы получим соотношение в алгебре $\mathrm{Z}_{n}$.

Назовем обрезанием результат этой процедуры получения соотношений в алгебре $\mathrm{Z}_{n}$ из соотношений в алгебре $\mathrm{Z}_{n+1}$ (при сформулированных выше условиях). Тогда все соотношения в алгебре $\mathrm{Z}_{n}$ могут быть получены обрезанием подходящих соотношений в алгебре $\mathrm{Z}_{n+1}$ •

Более того, каждое соотношение в алгебре $\mathrm{Z}_{n}$ единственным образом продолжается до соотношения в алгебре $\mathrm{Z}_{n+1}$, из которого оно же может быть получено процедурой обрезания; другими словами, имеется взаимно однозначное соответствие между множеством соотношений в алгебре $\mathrm{Z}_{n}$ и множеством тех соотношений в алгебре $\mathrm{Z}_{n+1}$, которые не содержат слагаемых, имеющих $z_{i, n+1}, i=1, \ldots, n$, в качестве левого сомножителя. 
Правило стабилизации не есть изолированный феномен для алгебры $\mathfrak{g l} ;$ оно может быть обобщено на некоторые другие четверки алгебр, заменяющих представленные в следующей диаграмме:

$$
\mathfrak{g l}_{n} \overbrace{\mathfrak{g l}_{n+1}}^{\mathfrak{g l}_{n} \oplus \mathfrak{g l}_{n}} \nearrow^{\mathfrak{g l}_{n+1}} \oplus \mathfrak{g l}_{n+1} .
$$

\section{§4. Полнота соотношений}

4.1. Приведем вначале общие аргументы, доказывающие более слабую версию теоремы 3 , в которой кольцо $\overline{\mathrm{U}}(\mathfrak{h})$ расширено до поля $\mathscr{D}(\mathfrak{h})$.

Как и выше, символом $\mathfrak{R}$ мы обозначаем систему соотношений (3.25)-(3.27), (3.29)-(3.31). Символом $\mathfrak{R}^{\prec}$ обозначим множество (3.10). Мы увидим, что система $\mathfrak{R}$ эквивалентна системе $\mathfrak{R}^{\prec}$. Система $\mathfrak{R}$ получается из соотношений (3.10), поскольку последние составляют множество определяющих соотношений для весовых образующих; остается вывести обратную импликацию. Обозначим временно образующие из множества $\left\{\check{z}_{i j},{ }_{i}\right\}$ символами $\widetilde{p}_{L}$, занумерованными лишь одним индексом $L, L=1, \ldots, n^{2}$. Число правил упорядочивания для $n^{2}$ переменных $\widetilde{p}_{L}$ равно $n^{2}\left(n^{2}-1\right) / 2$. Поэтому для доказательства полноты достаточно показать, что размерность (над $\mathscr{D}(\mathfrak{h}))$ подпространства, порожденного элементами системы $\mathfrak{R}$, не меньше $n^{2}\left(n^{2}-1\right) / 2$. Всякое соотношение из множества $\mathfrak{R}$ есть сумма произведений $\widetilde{p}_{L} \diamond \widetilde{p}_{M}$ с коэффициентами из $\overline{\mathrm{U}}(\mathfrak{h})$ плюс, быть может, слагаемое нулевой по элементам из $\widetilde{p}$ степени. Обозначим через $\mathfrak{R}_{0}$ систему $\mathfrak{R}$ с опущенными слагаемыми нулевой по элементам из $\widetilde{p}$ степени. Достаточно показать, что

\section{система $\mathfrak{R}_{0}$ содержит $n^{2}\left(n^{2}-1\right) / 2$} линейно независимых над $\mathscr{D}(\mathfrak{h})$ соотношений.

Переставив все коэффициенты из $\overline{\mathrm{U}}(\mathfrak{h})$ во всех соотношениях из $\mathfrak{R}_{0}$ в одну (скажем, правую) сторону от мономов $\widetilde{p}_{L} \diamond \widetilde{p}_{M}$, можно придать переменным $\stackrel{\circ}{h j}_{i j}$ произвольные численные значения (учитывая соотношения линейной зависимости между ними). Для проверки утверждения (4.1) достаточно найти такое множество значений переменных $\stackrel{\circ}{h}_{i j}$, для которого соответствующая система с числовыми коэффициентами содержит $n^{2}\left(n^{2}-1\right) / 2$ линейно независимых соотношений. Однако при $\stackrel{\circ}{h}_{i j}$, стремящихся к $\infty$ (следующим образом: $\stackrel{\circ}{h}_{i, i+1}=c_{i, i+1} h$, $h \rightarrow \infty$, где $c_{i, i+1}$ - ненулевые постоянные), непосредственное наблюдение показывает, что система $\mathfrak{R}_{0}$ превращается в систему $\widetilde{p}_{L} \diamond \widetilde{p}_{M}=\widetilde{p}_{M} \diamond \widetilde{p}_{L}, M>L$. Это доказывает полноту системы над полем $\mathscr{D}(\mathfrak{h})$.

Отметим, что приведенные выше аргументы не использовали согласованность порядка $\prec$ с частичным порядком $<$ на пространстве $\mathfrak{h}^{*}$.

4.2. Для заданного порядка пусть $X$ обозначает формальный вектор всех неупорядоченных произведений вида $\widetilde{p}_{L} \diamond \widetilde{p}_{K}$, а $Y$ - формальный вектор всех упорядоченных произведений. Для того чтобы переписать систему $\mathfrak{R}$ в форме упорядочивающих соотношений, необходимо разрешить для вектора $X$ систему линейных уравнений

$$
\mathscr{A} X=\mathscr{B} Y+C,
$$


где $C$ - вектор слагаемых степени 0 , а $\mathscr{A}$ и $\mathscr{B}$ - некоторые матрицы с коэффициентами из $\overline{\mathrm{U}}(\mathfrak{h})$ (согласно приведенному выше доказательству, $\mathscr{A}-$ квадратная матрица). Решение этой системы может привести к возникновению коэффициентов из $\mathscr{D}(\mathfrak{h})$ (а не из $\overline{\mathrm{U}}(\mathfrak{h}))$ в упорядочивающих соотношениях. Такое случается, например, для лексикографического порядка для образующих $z_{i j}$ и $t_{i}$ (здесь $z_{i i}=t_{i}$ ) алгебры $\mathrm{Z}_{n}$ при $n>2$ (мы не приводим деталей; это результат прямых вычислений). Из формулы (2.11) (и утверждения (е) §2) следует, что для порядка (3.8)-(3.9) решение системы (4.2) определено над кольцом $\overline{\mathrm{U}}(\mathfrak{h})$. Однако это говорит лишь о том, что для этого порядка возможные члены из $\mathscr{D}(\mathfrak{h})$ в определителе матрицы $\mathscr{A}$ сокращаются в комбинациях $\mathscr{A}^{-1} \mathscr{B}$ и $\mathscr{A}^{-1} C ;$ системы $\mathfrak{R}$ и $\mathfrak{R} \prec$ могут, тем не менее, не быть эквивалентными над $\overline{\mathrm{U}}(\mathfrak{h})$ в том смысле, что элементы матрицы $\mathscr{A}^{-1}$ могут не лежать в кольце $\overline{\mathrm{U}}(\mathfrak{h})$ и мы не можем перейти от системы $\mathfrak{R}$ к системе $\mathfrak{R} \prec$, составляя линейные над $\overline{\mathrm{U}}(\mathfrak{h})$ комбинации соотношений из $\mathfrak{R}$.

4.3. Перейдем теперь к доказательству теоремы 3. Пусть $\mathscr{F}-$ свободная алгебра над кольцом $\overline{\mathrm{U}}(\mathfrak{h})$ с весовыми образующими $z_{i j}$ и $t_{i}$. Обе системы, $\mathfrak{R}$ и $\mathfrak{R}$, определены над кольцом $\overline{\mathrm{U}}(\mathfrak{h})$, и имеет место гомоморфизм $\varpi: \mathscr{F} / \mathfrak{R} \rightarrow \mathscr{F} / \mathfrak{R} \prec$.

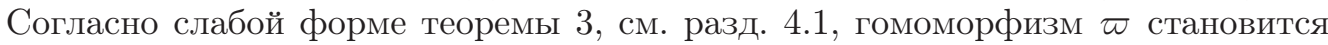
изоморфизмом после тензорного умножения на поле $\mathscr{D}(\mathfrak{h})$ (над кольцом $\overline{\mathrm{U}}(\mathfrak{h}))$. Докажем, что и само отображение $\varpi$ есть изоморфизм.

Доказательство использует индукцию по $n$, закон стабилизации и вычисление некоторых определителей. При этом на каждом шагу исследуется структура появляющихся знаменателей. Индукция начинается с $n=1$ и алгебры $\mathrm{Z}_{1}$, для которой доказывать нечего.

В общем случае нам необходимо показать, что числитель определителя матрицы $\mathscr{A}$, фигурирующей в равенстве (4.2), есть произведение линейных сомножителей вида (3.6). Поскольку соотношения весовые, матрица $\mathscr{A}$ имеет блочную структуру, ее блоки $\mathscr{A}_{\lambda}$ занумерованы весами соотношений. Определитель матрицы $\mathscr{A}$ есть произведение определителей блоков $\mathscr{A} \lambda$.

Так же, как и в разд. 3.4, рассмотрим алгебру $\mathrm{Z}_{n-1}$ как подпространство пространства $\mathrm{Z}_{n}$. Фиксируем вес $\lambda$ для алгебры $\mathrm{Z}_{n-1}$. Обозначим через $\mathscr{L}_{\lambda}^{(n)}$ линейную подсистему $\mathscr{A}_{\lambda} X_{\lambda}=\mathscr{B}_{\lambda} Y_{\lambda}+C_{\lambda}$ системы (4.2), соответствующую весу $\lambda$ алгебры $\mathrm{Z}_{n}$. Система $\mathscr{L}_{\lambda}^{(n)}$ содержит подсистему ${ }^{(n)} \mathscr{L}_{\lambda}^{(n-1)}$, соответствующую образующим из $\mathrm{Z}_{n-1}$ (структура предельных соотношений, построенных в разд. 4.1, показывает, что соотношения пронумерованы парами образующих, так что подсистема ${ }^{(n)} \mathscr{L}_{\lambda}^{(n-1)}$ корректно определена). Сравним ${ }^{(n)} \mathscr{L}_{\lambda}^{(n-1)}$ с соответствующей системой $\mathscr{L}_{\lambda}^{(n-1)}$ для алгебры $\mathrm{Z}_{n-1}$. Согласно принципу стабилизации, система $\mathscr{L}_{\lambda}^{(n-1)}$ получается из системы ${ }^{(n)} \mathscr{L}_{\lambda}^{(n-1)}$ процедурой обрезания в смысле разд. 3.4: между двумя системами имеется взаимно однозначное соответствие, при этом соотношения из системы ${ }^{(n)} \mathscr{L}_{\lambda}^{(n-1)}$ содержат дополнительные по сравнению с системой $\mathscr{L}_{\lambda}^{(n-1)}$ члены, в которые входят произведения $z_{n i} \diamond z_{j n}$ для некоторых $i, j<n$. В силу предположения индукции можно считать, что система $\mathscr{L}_{\lambda}^{(n-1)}$ эквивалентна над своим собственным кольцом $\overline{\mathrm{U}}(\mathfrak{h})$ системе упорядочивающих соотношений. Применение этих же преобразований 
к системе ${ }^{(n)} \mathscr{L}_{\lambda}^{(n-1)}$ также приводит к упорядочивающим соотношениям, поскольку слагаемые $z_{n i} \diamond z_{j n}$ уже упорядочены. Эти рассуждения показывают, что достаточно рассмотреть только подмножество соотношений, занумерованных образующими $\left(\widetilde{p}_{L}, \widetilde{p}_{M}\right)$, для которых

$$
\widetilde{p}_{L} \notin \mathrm{Z}_{n-1} \quad \text { или } \quad \widetilde{p}_{M} \notin \mathrm{Z}_{n-1} .
$$

Применяя построенные правила упорядочивания (эквивалентные системе $\left.{ }^{(n)} \mathscr{L}_{\lambda}^{(n-1)}\right)$ к этим оставшимся соотношениям, мы оставим в них из произведений $\widetilde{p}_{U} \widetilde{p}_{V}$, где $\widetilde{p}_{U}$ и $\widetilde{p}_{V} \in \mathrm{Z}_{n-1}$, только упорядоченные члены $\widetilde{p}_{L^{\prime}} \widetilde{p}_{M^{\prime}}, L^{\prime}<M^{\prime}$, c $\widetilde{p}_{L^{\prime}}, \widetilde{p}_{M^{\prime}} \in \mathrm{Z}_{n-1}$.

Нам осталось проследить за тем, что происходит с членами (весовых) соотношений, содержащими базисные элементы, не принадлежащие $\mathrm{Z}_{n-1}$. Рассмотрим отдельно каждый тип весовых соотношений, следуя нумерации, описанной в начале разд. 3.3. Соотношения типов 1 и 2 не доставляют трудностей.

Количество соотношений типов 3 и 4 увеличивается с ростом $n$. Замена переменных (3.18), а также перенормировка (3.24) и обратная к ней имеют допустимые знаменатели; поэтому мы можем работать с образующими $\check{t}_{i}$ и $\check{z}_{i j}$ вместо $t_{i}$ и $z_{i j}$.

4.4. Соотношения типа 4. Для соотношений (3.30) и (3.31) условие (4.3) означает, что в рассмотрении нуждается только подсистема соотношений (3.31), соответствующих парам $\left(z_{n i}, z_{i n}\right), i<n$. Следуя аргументам предыдущего раздела, мы предполагаем, что неупорядоченные мономы в этой подсистеме суть произведения $\stackrel{\circ}{z}_{i n} \diamond \stackrel{\circ}{z}_{n i}, i<n$. Перепишем подсистему в виде (4.2):

$$
\stackrel{\circ}{z}_{i n} \diamond \stackrel{\check{z}}{n i}_{a: a<n, a \neq i} \frac{1}{\stackrel{\circ}{h}_{i a}+1} \stackrel{\circ}{z}_{a n} \diamond \stackrel{\circ}{z}_{n a}=\ldots,
$$

где точками обозначены упорядоченные члены. Мы видим, что элементы мат-

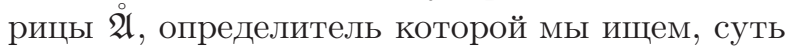

$$
\stackrel{\mathfrak{A}}{i j}_{i}:=\frac{1}{\stackrel{\circ}{h}_{i j}+1},
$$

где, напомним, $\stackrel{\circ}{h}_{i j}=\stackrel{\circ}{h}_{i}-\stackrel{\circ}{h}_{j}$; в частности, $\stackrel{\circ}{h}_{i i}=0$. Определитель этой матрицы хорошо известен. Матрица $\mathfrak{A}$ есть специализация матрицы $\mathfrak{A}$ с элементами

$$
\mathfrak{A}_{i j}:=\frac{1}{x_{i}+y_{j}}
$$

в точках $x_{i}=\stackrel{\circ}{h}_{i}$ и $y_{j}=-\stackrel{\circ}{h}_{j}+1$. Определитель матрицы $\mathfrak{A}$ вычислен в работе [3]: $\operatorname{det} \mathfrak{A}=\prod_{i, j: i<j}\left(\left(x_{i}-x_{j}\right)\left(y_{i}-y_{j}\right)\right) / \prod_{i, j}\left(x_{i}+y_{j}\right)$. Поэтому $\operatorname{det} \mathfrak{A}=$ $\prod_{i, j: i<j} \stackrel{\circ}{h}_{i j}^{2} /\left(\stackrel{\circ}{h}_{i j}^{2}-1\right)$. Таким образом, матрица, обратная к $\mathfrak{A}$, имеет допустимые знаменатели.

4.5. Соотношения типа 3. Для соотношений (3.27) и (3.29) типа 3 условие (4.3) означает, что достаточно исследовать лишь соотношения с весами $\varepsilon_{i}-\varepsilon_{n}$ и $\varepsilon_{n}-\varepsilon_{i}, i<n$. 
Начнем с веса $\varepsilon_{i}-\varepsilon_{n}$ при фиксированном $i, i<n$. Неупорядоченные квадратичные мономы веса $\varepsilon_{i}-\varepsilon_{n}$ суть

$$
\begin{aligned}
& \check{z}_{a n} \diamond \stackrel{\check{z}}{i a}_{i a}, \quad \text { где } a \text { таково, что } 2 a<i+n, a \neq i, \\
& \check{z}_{i j} \diamond \stackrel{\circ}{j}_{j n}, \quad \text { где } j \text { таково, что } i+n \leqslant 2 j, \\
& \check{z}_{i n} \diamond \dot{\circ}_{b} .
\end{aligned}
$$

В нашей системе участвуют все соотношения (3.27) и (3.29). Однако система имеет блочно-треугольную структуру, поддающуюся анализу.

Обозначим через $\mathfrak{r}_{i k l}$ то соотношение из списка $(3.27)$, у которого левая часть начинается с произведения $\stackrel{\circ}{i k}_{i k} \check{z}_{k l}$. Пусть $\kappa_{i k l}-$ коэффициент при произведении $\stackrel{\circ}{z}_{i k} \diamond \stackrel{\circ}{z}_{k l}$ в соотношении $\mathfrak{r}_{i k l}$. Соотношение $\mathfrak{r}_{i j n}$ можно записать в форме

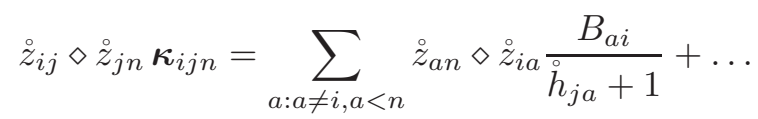

(поскольку $\stackrel{\circ}{h}_{j j}=0$ ). Здесь многоточие означает упорядоченные члены вида $\stackrel{\circ}{t}_{b} \diamond \dot{z}_{i n}$ (слагаемое $\stackrel{\circ}{z}_{j n} \diamond \dot{z}_{i j}$ включено в сумму перед многоточием). Среди неупорядоченных мономов (4.7)-(4.9) только мономы (4.7) встречаются в соотношениях $\mathfrak{r}_{i j n}$. При этом соответствующий индекс $j$ таков, что $2 j<i+n$ и $j \neq i$. Таким образом, подсистема $\left\{\mathfrak{r}_{i j n} \mid 2 j<i+n, j \neq i\right\}$ содержит столько же соотношений, сколько и неупорядоченных мономов. Элементы матрицы, определитель которой необходимо при этом вычислить в процессе выражения неупорядоченных мономов через упорядоченные - это $\mathfrak{A}_{a j}^{\prime}:=B_{a i} /\left(\stackrel{\circ}{h}_{j a}+1\right)$; ее $a$-й столбец содержит элемент $B_{a i}$ в качестве общего сомножителя, так что определитель матрицы $\mathfrak{A}^{\prime}$ есть произведение элементов $B_{a i}$ (по $a$, таким, что $2 a<i+n$ и $a \neq i)$, умноженное на определитель матрицы того же вида (4.5), что и выше. Таким образом, определитель матрицы $\mathfrak{A}^{\prime}$ обратим в кольце $\overline{\mathrm{U}}(\mathfrak{h})$. Мы используем указанную подсистему для упорядочивания мономов (4.7).

После упорядочивания мономов (4.7) оставшиеся соотношения $\mathfrak{r}_{i j n}$ (где $j$ таково, что $i+n \leqslant 2 j)$ превращаются в множество упорядочивающих соотношений для мономов (4.8); каждое соотношение содержит теперь в точности один неупорядоченный моном вида (4.8) с коэффициентом $\boldsymbol{\kappa}_{i j n}$, обратный к которому имеет допустимые знаменатели.

Множество соотношений (3.29) дает правила упорядочивания для мономов $\stackrel{\circ}{z}_{i n} \diamond \stackrel{\circ}{t}_{k}$ при условии, что упорядоченные выражения для всех мономов $\stackrel{\circ}{z}_{a n} \diamond \stackrel{\circ}{z}_{i a}$ уже известны.

4.6. Соотношения типа 3 веса $\varepsilon_{n}-\varepsilon_{\boldsymbol{i}}$. Рассмотрения разд. 4.2 показывают, что упорядочивающие соотношения (2.11) для любых двух порядков на весовом базисе пространства $\mathfrak{p}$, согласованных с частичным порядком < на пространстве $\mathfrak{h}^{*}$, эквивалентны над $\overline{\mathrm{U}}(\mathfrak{h})$. Определим вместо порядка (3.8)-(3.9) порядок ґ следующим образом:

$$
z_{i j} \dot{2} z_{k l}, \quad \text { если } i-j>k-l \text { или } \begin{cases}i>k, & \text { если } i-j=k-l>0, \\ i<k, & \text { если } i-j=k-l<0, \\ \text { как угодно, } & \text { если } i-j=k-l=0 .\end{cases}
$$


Особенность порядка 之 состоит в том, что антиинволюция $\epsilon$, см. (3.12), переводит множество упорядоченных квадратичных мономов произвольного ненулевого веса $\lambda$ в множество упорядоченных квадратичных мономов веса $-\lambda$.

В работе [5] доказано, что система $\mathfrak{R}$ замкнута относительно антиинволюции $\epsilon$ (т. е. системы $\mathfrak{R}$ и $\epsilon(\mathfrak{R})$ эквивалентны над $\overline{\mathrm{U}}(\mathfrak{h}))$. Для порядка 之 применение антиинволюции $\epsilon$ сводит вопрос об эквивалентности над кольцом $\overline{\mathrm{U}}(\mathfrak{h})$ множества соотношений $\mathfrak{R}$ и множества (3.10) правил упорядочивания веса $\varepsilon_{n}-\varepsilon_{i} \mathrm{~K}$ тому же вопросу для веса $\varepsilon_{i}-\varepsilon_{n}$. Из результатов предыдущего раздела следует

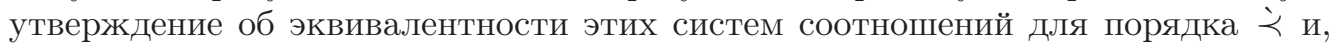
значит, для любого другого порядка, согласованного с частичным порядком < на множестве $\mathfrak{h}^{*}$, например, для порядка $\prec$.

Доказательство теоремы 3 завершено.

Множество $\mathfrak{R}^{\prec}$ упорядочивающих соотношений (3.10) по своей конструкции замкнуто над $\overline{\mathrm{U}}(\mathfrak{h})$ относительно инволюции $\omega$, см. формулу (3.13). В качестве приложения эквивалентности систем соотношений $\mathfrak{R}$ и $\mathfrak{R}^{\prec}$ над кольцом $\overline{\mathrm{U}}(\mathfrak{h})$ мы получаем утверждение о замкнутости над $\overline{\mathrm{U}}(\mathfrak{h})$ системы $\mathfrak{R}$ относительно инволюции $\omega$.

Отметим также, что все знаменатели, появляющиеся в доказательстве, имеют вид $\stackrel{\circ}{h}_{i j} \pm \varsigma, i<j$, где $\varsigma=0,1$ или 2.

4.7. Как показывает доказательство, по существу единственная матрица, которую необходимо обращать - это матрица $\mathfrak{A}$ с элементами вида (4.5). Элементы обратной к ней матрицы имеют вид

$$
\left(\stackrel{\mathfrak{A}}{ }^{-1}\right)_{i j}=-\frac{1}{\stackrel{\circ}{h j}_{i j}} \prod_{a: a \neq i} \frac{\stackrel{\circ}{i a}_{i a}-1}{\stackrel{\circ}{h}_{i a}} \prod_{b: b \neq j} \frac{\stackrel{\circ}{h b}_{j b}+1}{\stackrel{\circ}{h}_{j b}} .
$$

Проверка формулы (4.12) в форме равенства $\sum_{j}\left(\stackrel{\mathfrak{A}}{ }^{-1}\right)_{i j} \mathfrak{A}_{j k}=\delta_{j k}$, где $\delta_{j k}-$ символ Кронекера, сводится к тождеству

$$
\frac{1}{\stackrel{\circ}{h}_{i k}+1} \prod_{b: b \neq i} \frac{\stackrel{\circ}{h}_{i b}+1}{\stackrel{\circ}{h}_{i b}}-\sum_{j: j \neq i} \frac{1}{\stackrel{\circ}{h}_{i j}\left(\stackrel{\circ}{h}_{j k}+1\right)} \prod_{b: b \neq i, j} \frac{\stackrel{\circ}{h b}_{j b}+1}{\stackrel{\circ}{h}_{j b}}=\delta_{i k} \prod_{b: b \neq i} \frac{\stackrel{\circ}{h}_{i b}}{\stackrel{h}{i b}_{i b}-1},
$$

которое проверяется подсчетом вычетов и значения в бесконечности обеих частей равенства как функций переменной $\stackrel{\circ}{h}_{i}$.

Обратная к более общей матрице $\mathfrak{A}$ с элементами (4.6) имеет элементы вида

$$
\left(\mathfrak{A}^{-1}\right)_{i j}=\left(x_{j}+y_{i}\right) \prod_{a: a \neq j} \frac{x_{a}+y_{i}}{x_{a}-x_{j}} \prod_{b: b \neq i} \frac{y_{b}+x_{j}}{y_{b}-y_{i}} .
$$

Эта формула проверяется аналогично формуле (4.12) при помощи вычисления подходящих вычетов и значений в бесконечности.

Формула (4.12) эквивалентна специализации формулы (4.14) при $x_{i}=\stackrel{\circ}{h}_{i}$ и $y_{j}=-\stackrel{\circ}{h}_{j}+1$ (но не совпадает с ней буквально).

Формула (4.12) предоставляет рекурсивную процедуру преобразования системы $\mathfrak{R}$ в систему упорядочивающих соотношений.

Благодарности. Мы благодарим Лоика Пулэна д’Андеси за независимое подтверждение корректности соотношений разд. 3.3 посредством проверки 
(с использованием компьютера) теоремы Пуанкаре-Биркгофа-Витта для алгебры $\mathrm{Z}_{4}$, заданной образующими и соотношениями. Мы признательны Елене Огиевецкой за помощь в подготовке текста статьи.

Часть настоящей работы была выполнена во время визитов второго автора в CPT и CIRM в Марселе. Авторы благодарят сотрудников Институтов за предоставление прекрасных условий для работы во время этих визитов.

\section{ЛитерАТУРА}

[1] Р. М. Ашерова, Ю. Ф. Смирнов, В. Н. Толстой, Проекционные операторъ для простых групп Ли. II. Общая схема построения понижающих операторов. Группь $S U(n)$, ТМФ, 15:1 (1973), 107-119.

[2] Р. М. Ашерова, Ю. Ф. Смирнов, В.Н.Толстой, Описание некоторого класса проекиионных операторов для полупростых комплексных алгебр Ли, Матем. заметки, 26:1 (1979), 15-25.

[3] A. Cauchy, Mémoire sur les fonctions alternées et sur les sommes alternées, in: "Exercices d'analyse et de physique mathématique", Vol. 2, Bachelier, Paris, 1841, 151-159.

[4] S. Khoroshkin, O. Ogievetsky, Mickelsson algebras and Zhelobenko operators, J. Algebra, 319:5 (2008), 2113-2165.

[5] S. Khoroshkin, O. Ogievetsky, Structure constants of diagonal reduction algebras of $\mathfrak{g l}$ type (готовится к публикации).

[6] S. Khoroshkin, O. Ogievetsky, Examples of diagonal reduction algebras (готовится к публикации) .

[7] J. Mickelsson, Step algebras of semisimple subalgebras of Lie algebras, Rep. Math. Phys., 4:4 (1973), 307-318.

[8] Д. Желобенко, Представления редуктивных алгебр Ли, Наука, М., 1994.

Физический институт РАН,

Centre de Physique Théorique, Marseille

e-mail: oleg.ogievetsky@gmail.com

Институт теоретической и экспериментальной физики

e-mail: khor@itep.ru
Поступило в редакцию 29 июня 2009 г. 